January 28, 2012 11:14 WSPC/INSTRUCTION FILE crt unaryfa

International Journal of Algebra and Computation

(C) World Scientific Publishing Company

\title{
Unary FA-presentable semigroups
}

\author{
Alan J. Cain \\ Centro de Matemática, Universidade do Porto, \\ Rua do Campo Alegre 687, 4169-007 Porto, Portugal \\ ajcain@fc.up.pt \\ Nik Ruškuc \\ School of Mathematics and Statistics, University of St Andrews, \\ North Haugh, St Andrews, Fife KY16 9SS, United Kingdom \\ nik@mcs.st-andrews.ac.uk \\ Richard M. Thomas \\ Department of Computer Science, University of Leicester, \\ University Road, Leicester, LE1 7RH, United Kingdom \\ rmt@mcs.le.ac.uk \\ Received (Day Month Year) \\ Revised (Day Month Year) \\ Communicated by [editor]
}

\begin{abstract}
Automatic presentations, also called FA-presentations, were introduced to extend finite model theory to infinite structures whilst retaining the solubility of interesting decision problems. A particular focus of research has been the classification of those structures of some species that admit automatic presentations. Whilst some successes have been obtained, this appears to be a difficult problem in general. A restricted problem, also of significant interest, is to ask this question for unary automatic presentations: automatic presentations over a one-letter alphabet. This paper studies unary FA-presentable semigroups.

We prove the following: Every unary FA-presentable structure admits an injective unary automatic presentation where the language of representatives consists of every word over a one-letter alphabet. Unary FA-presentable semigroups are locally finite, but non-finitely generated unary FA-presentable semigroups may be infinite. Every unary FApresentable semigroup satisfies some Burnside identity. We describe the Green's relations in unary FA-presentable semigroups. We investigate the relationship between the class of unary FA-presentable semigroups and various semigroup constructions. A classification is given of the unary FA-presentable completely simple semigroups.
\end{abstract}

Keywords: Automatic presentations; semigroups; regular languages.

Mathematics Subject Classification 2010: 03D05, 20M35, 68Q45. 


\section{Introduction}

Automatic presentations, also known as FA-presentations, were introduced by Khoussainov \& Nerode [10] to fulfill a need to extend finite model theory to infinite structures while retaining the solubility of interesting decision problems, and have recently been applied to algebraic structures such as groups [14], rings [13], and semigroups $[2,3]$.

One main avenue of research has been the classification of those structures of some species that admit automatic presentations. Classifications are known for finitely generated groups [14, Theorem 8] and cancellative semigroups [3, Theorem 13], for integral domains (and more generally for possibly non-commutative rings with identity and no zero divisors) [13, Corollary 17], for Boolean algebras [11, Theorem 3.4], and for ordinals [7].

In several areas where general classifications remain elusive, it has been possible to classify those structures that admit unary automatic presentations (that is, automatic presentations over a one-letter alphabet), including, for example, bijective functions [1, Theorem 7.12], equivalence relations [1, Theorem 7.13] (see also Theorem 8 below), linear orders [1, Theorem 7.15], graphs [1, Theorem 7.16], and groups [1, Theorem 7.19]. (Notice that a classification result in the non-unary case is only known for finitely generated groups.) Furthermore, the isomorphism problem is decidable for certain unary FA-presentable structures [12].

This motivates the study of semigroups admitting unary automatic presentations, which forms the subject of this paper. Whilst we do not give a complete classification of such semigroups, we do describe a number of their properties, which lead to classifications in some special cases.

First, we prove a useful preliminary result that applies to all unary FA-presentable structures, not just to semigroups (Theorem 9). Example 11 shows that infinite unary FA-presentable semigroups exist, contrasting the fact that unary FA-presentable groups are finite. However, the first main result of the paper, that unary FA-presentable semigroups are locally finite (Theorem 13), yields the immediate corollary that finitely generated unary FA-presentable semigroups are finite (Corollary 14). Another consequence is that for any unary FA-presentable semigroup $S$, there exists some $n \in \mathbb{N}$ such that $S^{n+1}=S^{n}$.

Next, every unary FA-presentable semigroup is shown to satisfy some Burnside identity $x^{k}=x^{k+m}$ (Theorem 21), and therefore to be periodic. Consequently, the Green's relations $\mathcal{D}$ and $\mathcal{J}$ coincide in such semigroups. In Section 8, which focusses on the study of Green's relations for unary FA-presentable semigroups, it is proven that in such semigroups, $\mathcal{D}$-classes cannot contain both infinitely many $\mathcal{L}$-classes and infinitely many $\mathcal{R}$-classes. Furthermore, in a unary FA-presentable semigroup, there is a bound on the order of its $\mathcal{H}$-classes (Proposition 27).

Finally, Section 9 examines the interaction of the class of unary FA-presentable semigroups with extensions and subsemigroups, the Rees matrix construction, direct products, and free products. In particular, the results on Rees matrix semi- 
Unary FA-presentable semigroups 3

groups yield a classification of unary FA-presentable completely simple semigroups (Theorem 38).

\section{Preliminaries}

This section gathers the definitions and basic results needed elsewhere in the paper.

First of all, a terminological convention: throughout the paper, 'countable' means 'countably infinite'.

The reader is assumed to be familiar with the theory of finite automata and regular languages; see [8, Chs 2-3] for background reading. The empty word (over any alphabet) is denoted $\varepsilon$.

Definition 1. Let $L$ be a regular language over a finite alphabet A. Define, for $n \in \mathbb{N}$,

$$
L^{n}=\left\{\left(w_{1}, \ldots, w_{n}\right): w_{i} \in L \text { for } i=1, \ldots, n\right\} .
$$

Let $\$$ be a new symbol not in $A$. The mapping conv : $\left(A^{*}\right)^{n} \rightarrow\left((A \cup\{\$\})^{n}\right)^{*}$ is defined as follows. Suppose

$$
\begin{gathered}
w_{1}=w_{1,1} w_{1,2} \cdots w_{1, m_{1}}, \\
w_{2}=w_{2,1} w_{2,2} \cdots w_{2, m_{2}}, \\
\vdots \\
w_{n}=w_{n, 1} w_{n, 2} \cdots w_{n, m_{n}},
\end{gathered}
$$

where $w_{i, j} \in A$. Then $\operatorname{conv}\left(w_{1}, \ldots, w_{n}\right)$ is defined to be

$$
\left(w_{1,1}, w_{2,1}, \ldots, w_{n, 1}\right)\left(w_{1,2}, w_{2,2}, \ldots, w_{n, 2}\right) \cdots\left(w_{1, m}, w_{2, m}, \ldots, w_{n, m}\right),
$$

where $m=\max \left\{m_{i}: i=1, \ldots, n\right\}$ and with $w_{i, j}=\$$ whenever $j>m_{i}$.

Observe that the mapping conv maps an $n$-tuple of words to a word of $n$-tuples.

Definition 2. Let $A$ be a finite alphabet, and let $R \subseteq\left(A^{*}\right)^{n}$ be a relation on $A^{*}$. Then the relation $R$ is said to be regular if

$$
\operatorname{conv} R=\left\{\operatorname{conv}\left(w_{1}, \ldots, w_{n}\right):\left(w_{1}, \ldots, w_{n}\right) \in R\right\}
$$

is a regular language over $(A \cup\{\$\})^{n}$.

Definition 3. Let $\mathcal{S}=\left(S, R_{1}, \ldots, R_{n}\right)$ be a relational structure. Let $L$ be a regular language over a finite alphabet $A$, and let $\phi: L \rightarrow S$ be a surjective mapping. Then $(L, \phi)$ is an automatic presentation or an FA-presentation for $\mathcal{S}$ if:

(1) the relation $\Lambda(=, \phi)=\left\{\left(w_{1}, w_{2}\right) \in L^{2}: w_{1} \phi=w_{2} \phi\right\}$ is regular, and

(2) for each relation $R_{i}$ of arity $r_{i}$, the relation

$$
\Lambda\left(R_{i}, \phi\right)=\left\{\left(w_{1}, w_{2}, \ldots, w_{r_{i}}\right) \in L^{r_{i}}: R\left(w_{1} \phi, \ldots, w_{r_{i}} \phi\right)\right\}
$$

is regular. 
If $\mathcal{S}$ admits an automatic presentation, it is said to be FA-presentable.

If $(L, \phi)$ is an automatic presentation for $\mathcal{S}$ and the mapping $\phi$ is injective (so that every element of the structure has exactly one representative in $L)$, then $(L, \phi)$ is said to be injective.

If $(L, \phi)$ is an automatic presentation for $\mathcal{S}$ and $L$ is a language over a one-letter alphabet, then $(L, \phi)$ is a unary automatic presentation for $\mathcal{S}$, and $\mathcal{S}$ is said to be unary FA-presentable.

A semigroup can be viewed as a relational structure where the binary operation $\circ$ is interpreted as a ternary relation. The following definition simply restates that of a unary automatic presentation in the special case when the structure is a semigroup:

Definition 4. Let $S$ be a semigroup. Let $L$ be a regular language over the alphabet $\{a\}$, and let $\phi: L \rightarrow S$ be a surjective mapping. Then $(L, \phi)$ is a unary automatic presentation for $S$ if the relations

$$
\Lambda(=, \phi)=\left\{\left(w_{1}, w_{2}\right) \in L^{2}: w_{1} \phi=w_{2} \phi\right\}
$$

and

$$
\Lambda(\circ, \phi)=\left\{\left(w_{1}, w_{2}, w_{3}\right) \in L^{3}:\left(w_{1} \phi\right) \circ\left(w_{2} \phi\right)=w_{3} \phi\right\}
$$

are regular.

Often, the semigroup operation $\circ$ will be denoted simply by concatenation.

Proposition 5 ([10, Corollary 4.3]). Any structure that admits an automatic presentation $(L, \phi)$ admits an injective automatic presentation $\left(K,\left.\phi\right|_{K}\right)$, where $K \subseteq$ $L$.

Definition 6. If $(L, \phi)$, where $L \subseteq a^{*}$, is an injective unary automatic presentation for a structure $\mathcal{S}$, and $s$ is an element of $\mathcal{S}$, then $\ell(s)$ is the length of the unique word $w \in L$ with $w \phi=s$. [Notice that $a^{\ell(s)}=s \phi^{-1}$ for all elements $s$ of $\mathcal{S}$.]

The fact that a tuple of elements $\left(s_{1}, \ldots, s_{n}\right)$ of a structure $\mathcal{S}$ satisfies a firstorder formula $\theta\left(x_{1}, \ldots, x_{n}\right)$ is denoted $\mathcal{S} \models \theta\left(s_{1}, \ldots, s_{n}\right)$.

Proposition 7 ([10]). Let $\mathcal{S}$ be a structure with an automatic presentation $(L, \phi)$. For every first-order formula $\theta\left(x_{1}, \ldots, x_{n}\right)$ over the structure, the relation

$$
\Lambda(\theta, \phi)=\left\{\left(w_{1}, \ldots, w_{n}\right) \in L^{n}: \mathcal{S} \models \theta\left(w_{1} \phi, \ldots, w_{n} \phi\right)\right\}
$$

is regular.

Proposition 7 is fundamental to the theory of automatic presentations and will be used without explicit reference throughout the paper.

The following characterization of unary FA-presentable equivalence relations will be needed later:

Theorem 8 ([1, Theorem 7.13]). An equivalence relation $\sim$ is unary FA-presentable if and only if 
(1) the cardinality of the finite $\sim$-classes is bounded, and

(2) there are only finitely many countable $\sim$-classes.

For any subset $X$ of a semigroup $S$, denote by $X^{n}$ the set of all elements of $S$ that can be expressed as products of elements of $X$ of length exactly $n$ : that is, $X^{n}=\left\{x_{1} x_{2} \cdots x_{n}: x_{i} \in X\right\}$. Notice that in general $X^{n} \nsubseteq X^{n+1}$.

\section{General unary FA-presentable structures}

The following result shows that a unary FA-presentable structure admits an injective unary FA-presentation where the language of representatives is the language of all words over a one-letter alphabet. Observe that this result holds for all unary FApresentable structures, not just for semigroups.

Theorem 9. Let $\mathcal{S}$ be an infinite relational structure that admits a unary automatic presentation. Then $\mathcal{S}$ has an injective unary automatic presentation $\left(a^{*}, \psi\right)$.

Proof. By Proposition 5, assume without loss of generality, that $(L, \phi)$ is an injective unary automatic presentation for $\mathcal{S}$, where $L \subseteq b^{*}$.

Let $\mathfrak{B}$ be a deterministic complete finite automaton recognizing $L$. Suppose $\mathfrak{B}$ has state set $Q$, set of accept states $Y$, initial state $q_{0}$, and transition function $\delta: Q \times\{b\} \rightarrow Q$. Since the input alphabet has only one letter, each state has exactly one edge leaving it. Let $y_{1}, y_{2}, \ldots \in Y$ be the accept states in the order in which they are encountered when $\mathfrak{B}$ reads an arbitrarily long word over $\{b\}$. (The sequence of states $y_{i}$ is infinite because the language $L$ is infinite.) Let $\beta_{0}$ be the smallest non-negative integer such that $\left(q_{0}, b^{\beta_{0}}\right) \delta \in Y$, and for each $i \in \mathbb{N}$, let $\beta_{i}$ be the smallest positive integer such that $\left(y_{i}, b^{\beta_{i}}\right) \delta \in Y$. Notice that $\left(q_{0}, b^{\beta_{0}}\right) \delta=y_{1}$ and that $\left(y_{i}, b^{\beta_{i}}\right) \delta=y_{i+1}$. For $k \in \mathbb{N} \cup\{0\}$, let $B_{k}=\sum_{i=0}^{k} \beta_{i}$; notice that since $\beta_{i}>0$ for every $i \in \mathbb{N}$, the map $k \mapsto B_{k}$ is injective. Note that $\left(q_{0}, b^{B_{k}}\right) \delta=y_{k+1}$. Therefore the map $\psi$ from $a^{*}$ to the domain of $\mathcal{S}$ defined by $a^{k} \psi=b^{B_{k}} \phi$ is a bijection

Let $\mathcal{R}$ be some relation of $\mathcal{S}$ of arity $n$. (Possibly, $\mathcal{R}$ is the equality relation.) Let $\mathfrak{A}$ be an $n$-tape synchronous automaton recognizing $\operatorname{conv}(\Lambda(\mathcal{R}, \phi))$. Suppose that $\mathfrak{A}$ has state set $P$, initial state $p_{0}$, transition function $\zeta: P \times\{b, \$\}^{n} \rightarrow P$, and set of accept states $Z$.

Construct an $n$-tape synchronous automaton $\mathfrak{A}^{\prime}$ as follows. The state set is $P \times Y$, the inital state is $\left(p_{0}, y_{0}\right)$, the set of accept states is $Z \times Y$. The transition function $\kappa:(P \times Y) \times\{a, \$\}^{n} \rightarrow(P \times Y)$ is defined as follows:

$$
\left(\left(p, y_{i}\right),\left(a_{1}, \ldots, a_{n}\right)\right) \kappa=\left(\left(p,\left(v_{1}, \ldots, v_{n}\right)\right) \zeta, y_{i+1}\right),
$$

where

$$
v_{j}= \begin{cases}b^{\beta_{i}} & \text { if } a_{j}=a, \\ \$^{\beta_{i}} & \text { if } a_{j}=\$ .\end{cases}
$$

By construction, the new automaton $\mathfrak{A}^{\prime}$ accepts $\operatorname{conv}\left(a^{k_{1}}, \ldots, a^{k_{n}}\right)$ if and only if the original automaton $\mathfrak{A}$ accepts $\operatorname{conv}\left(b^{B_{k_{1}}}, \ldots, b^{B_{k_{n}}}\right)$. [In particular, note that $\mathfrak{A}$ 
can only accept $\operatorname{conv}\left(b^{h_{1}}, \ldots, b^{h_{n}}\right)$ if every $h_{i}$ is $B_{j_{i}}$ for some $j_{i}$, since $b^{h_{i}}$ must be accepted by $\mathfrak{B}$.] Thus, since $L(\mathfrak{A})=\operatorname{conv}(\Lambda(\mathcal{R}, \phi))$, it follows from the definition of $\psi$ that $L\left(\mathfrak{A}^{\prime}\right)=\operatorname{conv}(\Lambda(\mathcal{R}, \psi))$.

Since $\mathcal{R}$ was an arbitrary relation of $\mathcal{S}$, it follows that $\left(a^{*}, \psi\right)$ is a unary automatic presentation for $\mathcal{S}$.

\section{Finite groups and infinite semigroups}

The following result was first observed for groups Blumensath [1, Theorem 7.19]. Blumensath's proof generalizes immediately to cancellative semigroups [3, Theorem 12.1], although Theorem 9 could be used to give a more conceptually economical proof. In particular, Theorem 9 makes Blumensath's notion of 'loop constants' [1, Section 7.1] unnecessary for the proof.

Proposition 10. Unary FA-presentable cancellative semigroups are finite. In particular, unary FA-presentable groups are finite.

The following example shows that Proposition 10 does not extend to general semigroups, because infinite unary FA-presentable semigroups exist:

Example 11. Any countable right zero semigroup or left zero semigroup is unary FA-presentable. To see this, let $S=\left\{z_{i}: i \in \mathbb{N} \cup\{0\}\right\}$ be a countable right zero semigroup. (The reasoning for left zero semigroups is similar.)

Define $\phi: a^{*} \rightarrow S$ by $a^{n} \mapsto z_{n}$ for all $n \in \mathbb{N} \cup\{0\}$. Then

$$
\Lambda(=, \phi)=\left\{\left(a^{p}, a^{p}\right): p \in \mathbb{N} \cup\{0\}\right\}
$$

and

$$
\begin{aligned}
\Lambda(\circ, \phi) & =\left\{\left(a^{p}, a^{q}, a^{r}\right): a^{p} \phi \circ a^{q} \phi=a^{r} \phi, p, q, r \in \mathbb{N} \cup\{0\}\right\} \\
& =\left\{\left(a^{p}, a^{q}, a^{r}\right): z_{p} \circ z_{q}=z_{r}, p, q, r \in \mathbb{N} \cup\{0\}\right\} \\
& =\left\{\left(a^{p}, a^{q}, a^{r}\right): z_{q}=z_{r}, p, q, r \in \mathbb{N} \cup\{0\}\right\} \\
& =\left\{\left(a^{p}, a^{q}, a^{q}\right): p, q \in \mathbb{N} \cup\{0\}\right\},
\end{aligned}
$$

and so $\Lambda(=, \phi)$ and $\Lambda(\circ, \phi)$ are regular. Thus $\left(a^{*}, \phi\right)$ is a unary automatic presentation for $S$.

Note in passing that any finite semigroup - indeed, any finite structure admits a unary automatic presentation.

\section{Adjoining an identity}

Although the natural place for the following result would be in the discussion of semigroup constructions in Section 9, it is required in Section 6 and so is proved here instead:

Proposition 12. Let $S$ be a semigroup. Then $S$ is unary FA-presentable if and only if $S^{1}$ is unary FA-presentable. 
Proof. Suppose that $S$ admits an injective unary FA-presentation $\left(a^{*}, \phi\right)$. Define a new map $\psi: a^{*} \rightarrow S^{1}$ by $\varepsilon \psi=1$ and $(a w) \psi=w \phi$. [The idea is to lengthen all representatives by a single letter $a$ and use the empty word $\varepsilon$ to represent the adjoined identity.] Then $\psi$ is injective and

$$
\begin{aligned}
\Lambda(\circ, \psi)= & \left\{(u, v, w): u, v, w \in a^{*},(u \psi)(v \psi)=w \psi\right\} \\
= & \left\{(u, v, w): u, v, w \in a^{+},(u \psi)(v \psi)=w \psi\right\} \\
& \cup\left\{(u, \varepsilon, u),(\varepsilon, u, u): u \in a^{*}\right\} \\
= & \left\{\left(a u^{\prime}, a v^{\prime}, a w^{\prime}\right): u^{\prime}, v^{\prime}, w^{\prime} \in a^{+},\left(u^{\prime} \phi\right)\left(v^{\prime} \phi\right)=w^{\prime} \phi\right\} \\
& \cup\left\{(u, \varepsilon, u),(\varepsilon, u, u): u \in a^{*}\right\} \\
= & (a, a, a) \Lambda(\circ, \phi) \\
& \cup\left\{(u, \varepsilon, u),(\varepsilon, u, u): u \in a^{*}\right\},
\end{aligned}
$$

which is regular. So $\left(a^{*}, \psi\right)$ is an injective unary FA-presentation for $S^{1}$.

Suppose now that $\left(a^{*}, \phi\right)$ is an injective unary FA-presentation for $S^{1}$. Let $u \in$ $a^{*}$ be the unique word representing the adjoined identity. Then $a^{*}-\{u\}$ maps injectively onto $S$ and

$$
\Lambda\left(\circ,\left.\phi\right|_{a^{*}-\{u\}}\right)=\Lambda(\circ, \phi) \cap\left(\left(a^{*}-\{u\}\right) \times\left(a^{*}-\{u\}\right) \times\left(a^{*}-\{u\}\right)\right)
$$

is regular; hence $\left(a^{*}-\{u\},\left.\phi\right|_{a^{*}-\{u\}}\right)$ is a unary FA-presentation for $S$.

\section{Finitely generated unary FA-presentable semigroups}

While unary FA-presentable groups are finite by Proposition 10, Example 11 shows that unary FA-presentable semigroups may be infinite. However, with the extra condition of finite generation, finitude is guaranteed:

Theorem 13. Unary FA-presentable semigroups are locally finite.

Proof. Let $S$ be a unary FA-presentable semigroup. Let $Y$ be a finite subset of $S$. The aim is to show that the subsemigroup $T$ generated by $Y$ is finite.

By Proposition 12 and Theorem 9, $S^{1}$ admits an injective unary FA-presentation $\left(a^{*}, \phi\right)$. Let $X=Y \cup\{1\}$. Then $X$ generates the subsemigroup $T^{1}$ of $S^{1}$. Let $R=\max \{l(a): a \in X\}$. By [4, Lemma 7.5], there is a constant $N$ such that, for all $m \in \mathbb{N}$,

$$
\max \left\{\ell\left(a_{1} \cdots a_{m}\right): a_{i} \in X\right\} \leq R+\left\lceil\log _{2} m\right\rceil N .
$$

In a language over a one-letter alphabet, words are uniquely determined by their lengths. It thus follows from (12) that for all $m \in \mathbb{N}$,

$$
\left|X^{m}\right| \leq R+\left\lceil\log _{2} m\right\rceil N .
$$

Since $X$ contains the identity 1, it follows that $X^{m} \subseteq X^{m+1}$. So $\left|X^{m}\right| \leq\left|X^{m+1}\right|$.

Suppose that $\left|X^{m}\right|<\left|X^{m+1}\right|$ for all $m \in \mathbb{N}$. Then since $\left|X^{m}\right|$ must be an integer, $\left|X^{m}\right| \geq m$ for all $m \in \mathbb{N}$. Hence $m \leq R+\left\lceil\log _{2} m\right\rceil N$ for all $m \in \mathbb{N}$ by (13), which is 
a contradiction, for this inequality is false for sufficiently large $m$. Therefore there is some $m \in \mathbb{N}$ such that $\left|X^{m}\right|=\left|X^{m+1}\right|$.

So $X^{m}=X^{m+1}$. Hence $X^{m}$ contains all the elements of $X$ and is closed under right- and left-multiplication by elements of $X$. So $X^{m}$ must be the subsemigroup generated by $X$, which is $T^{1}$. Hence $T^{1}$ is finite and thus so is $T$.

Since $X$ was an arbitrary finite subset of the unary FA-presentable semigroup $S$, it follows that $S$ is locally finite.

Corollary 14. A finitely generated semigroup is unary FA-presentable if and only if it is finite.

Proof. In one direction, the result is obvious: if a semigroup is finite it admits a unary automatic presentation. In the other, it is a consequence of Theorem 13.

Notice that Corollary 14 gives a classification of those finitely generated semigroups that admit unary automatic presentations. Finite generation seems to be a useful tool for proving classification results for general (not just unary) FA-presentable structures; witness the classifications of finitely generated FA-presentable groups [14, Theorem 8] and finitely generated FA-presentable cancellative semigroups [3, Theorem 13].

Theorem 15. Let $S$ be a unary FA-presentable semigroup. Then there exists $n \in \mathbb{N}$ such that $S^{n+1}=S^{n}$.

Proof. First of all, notice that if $S$ is finite, the result holds trivially. Therefore assume without loss of generality that $S$ is infinite. Let $\left(a^{*}, \phi\right)$ be an injective unary FA-presentation for $S$.

Let $\leq$ be the $\mathcal{R}$ partial order, where

$$
x \leq y \Longleftrightarrow x=y \vee(\exists p)(x=y p) ;
$$

that is, $x \leq y$ if $x$ is a right-multiple of $y$. Since $\leq$ is first-order definable, $\operatorname{conv} \Lambda(\leq, \phi)$ is recognized by a deterministic finite automaton $\mathfrak{A}$.

Consider the structure of the automaton $\mathfrak{A}$. The path of edges labelled by $(a, a)$ starting at its initial state eventually enters a loop. (This loop is unique since $\mathfrak{A}$ is deterministic.) From any one of the states along this path, paths of edges labelled by $(a, \$)$ or by $(\$, a)$ may begin, each also leading into a uniquely determined loop. Let $t$ be a multiple of the lengths of each of these loops in $\mathfrak{A}$ and also greater than the number of states in $\mathfrak{A}$. Consider a word $\operatorname{conv}\left(a^{i}, a^{j}\right) \in L(\mathfrak{A})$. If $i$ and $j$ are greater than $t$, then in reading the prefix of this word consisting of symbols $(a, a)$, the automaton enters the first loop. Since $t$ is a multiple of the length of this loop, the word can be pumped so as to give words of the form $\operatorname{conv}\left(a^{i+h t}, a^{j+h t}\right)$. Similarly, if the difference between $i$ and $j$ is greater than $t$, the automaton enters a loop after the end of the prefix consisting of symbols $(a, a)$. Thus, since $t$ is a multiple of the length of this loop, the word can be pumped so as to give words of 
the form $\operatorname{conv}\left(a^{i}, a^{j+h t}\right)$ (in the case where $i+t \leq j$ ) or $\operatorname{conv}\left(a^{i+h t}, a^{j}\right)$ (in the case where $j+t \leq i)$. Therefore the following conditions hold:

(1) $\left(a^{i} \phi \leq a^{j} \phi\right) \wedge(i, j \geq t) \Longrightarrow(\forall h \in \mathbb{N})\left(a^{i+h t} \phi \leq a^{j+h t} \phi\right)$;

(2) $\left(a^{i} \phi \leq a^{j} \phi\right) \wedge(i+t \leq j) \Longrightarrow(\forall h \in \mathbb{N})\left(a^{i} \phi \leq a^{j+h t} \phi\right)$;

(3) $\left(a^{i} \phi \leq a^{j} \phi\right) \wedge(j+t \leq i) \Longrightarrow(\forall h \in \mathbb{N})\left(a^{i+h t} \phi \leq a^{j} \phi\right)$.

For $m \in\{0, \ldots, t-1\}$, let $A_{m}=\left\{a^{m+h t}: h \in \mathbb{N}\right\}$.

For each $k \in \mathbb{N}$, let $D_{k}=S^{k}-S^{k+1}$. Then each set $D_{k}$ consists of those elements of $S$ that can be written as a product of length $k$ but not of length $k+1$.

Notice that if $x, y \in D_{k}$ and $x \neq y$ then $x$ and $y$ are $\leq$-incomparable, for otherwise $x$ is a right multiple of $y$ or vice versa. Thus $x$ could be expressed as a longer product than $y$ or vice versa, contradicting the definition of $D_{k}$.

The following technical lemma concerns the relationship between subproducts and the various $D_{k}$ :

Lemma 16. If $s_{1} s_{2} \cdots s_{k} \in D_{k}$, then every subproduct $s_{i} s_{i+1} \cdots s_{j}$ belongs to $D_{j-i+1}$.

Proof. If the product $s_{i+1} \cdots s_{j}$ is equal to a longer product $t_{1} \cdots t_{h}$ (where $h>$ $i-j+1)$, then

$$
s_{1} s_{2} \cdots s_{k}=s_{1} \cdots s_{i-1} t_{1} \cdots t_{h} s_{j+1} \cdots s_{k},
$$

which is a product of more than $k$ elements of $S$ and so cannot lie in $D_{k}$. Hence $s_{i+1} \cdots s_{j}$ is not equal to any product of more than $j-i+1$ elements and so belongs to $D_{j-i+1}$.

We return to the proof of Theorem 15 .

Let $x \in D_{k}$. As a consequence of Lemma 16 , there is some $y \in D_{k-1}$ such that $x$ is a right multiple of $y$ and so $x<y$. Conversely, if $z \in S$ is such that $x<z$, then $x$ is a right-multiple of $z$ and so $z \in D_{k^{\prime}}$ for some $k^{\prime}<k$ (since otherwise $x$ could be expressed as a product of more than $k$ elements).

Suppose, with the aim of obtaining a contradiction, that infinitely many of the sets $D_{k}$ are infinite. Then since there are only finitely many sets $A_{m}$, there is some $m \in\{0, \ldots, t-1\}$ and such that $A_{m} \phi \cap D_{k}$ is infinite for some $k \in \mathbb{N}$. Call any such set $A_{m}$ good.

The following two lemmata concern the interaction between good sets and the relation $\leq$ :

Lemma 17. If $A_{m}$ is good, $a^{i} \in A_{m}, a^{j} \in A_{m^{\prime}}, i, j \geq t$, and $a^{i} \phi \leq a^{j} \phi$, then $A_{m^{\prime}}$ is good.

Proof. Suppose the opposite, that $A_{m^{\prime}}$ is not good. Then $m \neq m^{\prime}$ and thus $i \not \equiv j$ $\bmod t$. Let $k$ be such that $A_{m} \phi \cap D_{k}$ is infinite. Notice that $a^{i+h t} \in A_{m}$ for every $h \in \mathbb{N}$ by the definition of $A_{m}$. Since $A_{m} \phi \cap D_{k}$ is infinite, there are infinitely many 
$h \in \mathbb{N}$ such that $a^{i+h t} \phi \in D_{k}$. By condition (1) above, for all such $h$, the inequality $a^{i+h t} \phi<a^{j+h t}$ holds. Notice that $a^{j+h t} \in A_{m^{\prime}}$ for all such $h$. Furthermore, for each such $h$, the element $a^{j+h t} \phi$ is in $D_{k^{\prime}}$ for some $k^{\prime}<k$ since $a^{i+h t} \phi<a^{j+h t} \phi$. However, since $A_{m^{\prime}}$ is not good, $A_{m^{\prime}} \phi \cap D_{k^{\prime}}$ is finite. So there are only finitely many $h$ such that $a^{j+h t} \in A_{m^{\prime}}$ and $a^{j+h t} \phi \in D_{k^{\prime}}$ for $k^{\prime}<k$. This is a contradiction and so $A_{m^{\prime}}$ is good.

Lemma 18. There are arbitrarily long ascending chains of elements represented by words that have length greater than $t$ and that lie in good sets.

Proof. Let $h \in \mathbb{N}$. We will prove that there exists such an ascending chain of length $h$. Since infinitely many of the $D_{k}$ are infinite, there is some infinite $D_{k}$ with $k>h+t+1$. Since there are only $t$ distinct $A_{m}$, there is some $m \in\{0, \ldots, t-1\}$ such that $A_{m} \phi \cap D_{k}$ is infinite, so that $A_{m}$ is good. Choose $a^{i_{0}} \in A_{m}$ with $a^{i_{0}} \phi \in D_{k}$ and $\left|a^{i_{0}}\right|>t$.

Now proceed iteratively: Suppose that for $j \in\{0, \ldots, k-1\}$, we have a word $a^{i_{j}}$ with $a^{i_{j}} \phi \in D_{k-j}$. Then there is some $a^{i_{j+1}}$ with $a^{i_{j+1}} \phi \in D_{k-j-1}$ and $a^{i_{j+1}} \phi>$ $a^{i_{j}} \phi$.

This yields an ascending chain $a^{i_{0}} \phi<\ldots<a^{i_{k}} \phi$. Since all these elements are distinct, at most $t+1$ of the words $a^{i_{j}}$ have length at most $t$. Removing these elements gives an ascending chain of at least $h$ elements, all represented by words of length greater than $t$. Notice that the first element $a^{i_{0}} \phi$ is not removed, since it was chosen so that $a^{i_{0}}$ has length greater than $t$. Since $a^{i_{0}}$ lies in a good set, Lemma 17 now applies iteratively to show that all of the $a^{i_{j}}$ lie in good sets.

Again we return to the proof of Theorem 15 .

By Lemma 18, and since there are only $t$ distinct sets $A_{m}$, it is possible to choose $a^{i}$ and $a^{j}$ in some good set $A_{m}$ with $|i-j|>t$, both $i$ and $j$ greater than $t$, and $a^{i} \phi<a^{j} \phi$. Let $k$ be such that $A_{m} \phi \cap D_{k}$ is infinite. Assume that $i<j$; the case $i>j$ is similar. Choose $h$ with $a^{i+h t} \phi \in A_{m} \phi \cap D_{k}$. By condition (1) above, $a^{i+h t} \phi<a^{j+h t} \phi$. Choose $h^{\prime}$ with $a^{j+h t+h^{\prime} t} \in A_{m} \phi \cap D_{k}$. By condition (2) above, $a^{i+h t} \phi<a^{j+h t+h^{\prime} t} \phi$. This is a contradiction since $a^{i+h t} \phi, a^{j+h t+h^{\prime} t} \phi \in D_{k}$. Thus only finitely many of the $D_{k}$ are infinite.

So suppose $D_{k}$ is finite for every $k \geq r$. Every element of the set $D_{r} \cup D_{r+1} \cup \ldots$ can be written as a product of elements from $D_{r} \cup D_{r+1} \cup \ldots \cup D_{2 r-1}$ : just bracket the products in groups of $r$, each of which lies in $D_{r}$ by Lemma 16, except for the last one which has length between $r$ and $2 r-1$, which lies in $D_{r} \cup D_{r+1} \cup \ldots \cup D_{2 r-1}$ by Lemma 16 .

So the set $D_{r} \cup D_{r+1} \cup \ldots$ lies in the subsemigroup generated by $D_{r} \cup D_{r+1} \cup \ldots \cup$ $D_{2 r-1}$. But $D_{r} \cup D_{r+1} \cup \ldots \cup D_{2 r-1}$ is finite, and $S$ is locally finite by Theorem 13, so the set $D_{r} \cup D_{r+1} \cup \ldots$ is finite as well. Hence there exists $n \in \mathbb{N}$ such that $D_{n}=\emptyset$, and so $S^{n+1}=S^{n}$. 


\section{Burnside identities}

The present section is dedicated to proving that any unary FA-presentable semigroup satisfies some Burnside identity; that is, some semigroup identity $x^{k}=x^{k+m}$. (The constants $k, m \in \mathbb{N}$ are dependent on the semigroup in question.) In particular, any such semigroup is periodic and has bounded period.

First, two technical results are needed. The first restricts the length of the word representing a product of two elements in terms of the lengths of the words representing those elements themselves. In the language $a^{*}$, of course, the length of a word uniquely determines that word, so this restriction is very useful.

Lemma 19. Let $S$ be an infinite semigroup admitting an injective unary automatic presentation $\left(a^{*}, \phi\right)$ (by Theorem 9). Then there is a constant $n \in \mathbb{N}$ such that, for any $x, y \in S$, one of the following conditions holds:

(1) $\ell(x)-n \leq \ell(x y) \leq \ell(x)+n$,

(2) $\ell(y)-n \leq \ell(x y) \leq \ell(y)+n$,

(3) $\ell(x y) \leq n$.

Proof. Let $\mathfrak{A}$ be an automaton recognizing $\operatorname{conv} \Lambda(\circ, \phi)$ and let $n$ be the number of states in $\mathfrak{A}$.

Let $x, y \in S$. If $\ell(x y) \leq n$, then condition 3 holds and there is nothing to prove. So suppose $\ell(x y)>n$. Assume that $\ell(x) \leq \ell(y)$; the other case is similar. Suppose, with the aim of obtaining a contradiction, that neither condition 1 nor condition 2 holds. Then one of the following conditions holds:

$$
n<\ell(x y)<\ell(x)-n \text {, or } \ell(x)+n<\ell(x y)<\ell(y)-n \text {, or } \ell(x y)>\ell(y)+n \text {. }
$$

Each of the possible ranges for $\ell(x y)$ leads to a contradiction:

(1) $n<\ell(x y)<\ell(x)-n$. Then the following diagram describes the situation:

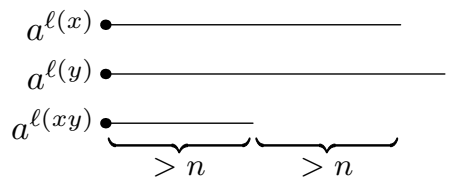

So the word $\operatorname{conv}\left(a^{\ell(x)}, a^{\ell(y)}, a^{\ell(x y)}\right)$ can be pumped before the end of $a^{\ell(x y)}$ and between the end of $a^{\ell(x y)}$ and the end of $a^{\ell(x)}$. That is, there exist $p, q \in \mathbb{N}$ with $0<p, q<n$ such that

$$
\left(a^{\ell(x)+i p+j q}, a^{\ell(y)+i p+j q}, a^{\ell(x y)+i p}\right) \in \Lambda(\circ, \phi)
$$

for all $i, j \in \mathbb{N} \cup\{0\}$. Setting $i=q$ and $j=0$ and then $i=0$ and $j=p$ shows that

$$
\left(a^{\ell(x)+q p}, a^{\ell(y)+q p}, a^{\ell(x y)+q p}\right),\left(a^{\ell(x)+p q}, a^{\ell(y)+p q}, a^{\ell(x y)}\right) \in \Lambda(\circ, \phi),
$$

which implies that $a^{\ell(x y)+q p} \phi=a^{\ell(x y)} \phi$, contradicting the injectivity of $\phi$. 
(2) $\ell(x)+n<\ell(x y)<\ell(y)-n$. Then the following diagram describes the situation:

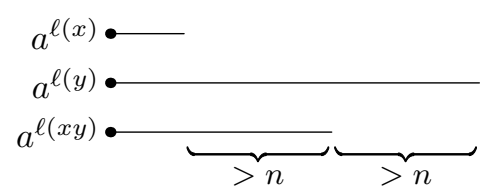

So the word $\operatorname{conv}\left(a^{\ell(x)}, a^{\ell(y)}, a^{\ell(x y)}\right)$ can be pumped between the end of $a^{\ell(x)}$ and the end of $a^{\ell(x y)}$ and between the end of $a^{\ell(x y)}$ and the end of $a^{\ell(y)}$. That is, there exist $p, q \in \mathbb{N}$ with $0<p, q<n$ such that

$$
\left(a^{\ell(x)}, a^{\ell(y)+i p+j q}, a^{\ell(x y)+i p}\right) \in \Lambda(\circ, \phi)
$$

for all $i, j \in \mathbb{N} \cup\{0\}$. Setting $i=q$ and $j=0$ and then $i=0$ and $j=p$ shows that

$$
\left(a^{\ell(x)}, a^{\ell(y)+q p}, a^{\ell(x y)+q p}\right),\left(a^{\ell(x)}, a^{\ell(y)+p q}, a^{\ell(x y)}\right) \in \Lambda(\circ, \phi),
$$

which implies that $a^{\ell(x y)+q p} \phi=a^{\ell(x y)} \phi$, contradicting the injectivity of $\phi$.

(3) $\ell(y)+n<\ell(x y)$. Then the following diagram describes the situation:

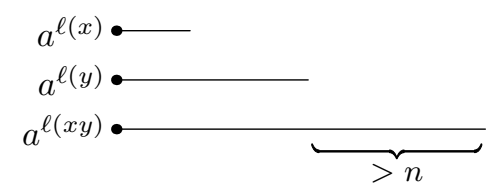

So the word $\operatorname{conv}\left(a^{\ell(x)}, a^{\ell(y)}, a^{\ell(x y)}\right)$ can be pumped between the end of $a^{\ell(y)}$ and the end of $a^{\ell(x y)}$. That is, there exists $p \in \mathbb{N}$ with $0<p<n$ such that

$$
\left(a^{\ell(x)}, a^{\ell(y)}, a^{\ell(x y)+i p}\right) \in \Lambda(\circ, \phi)
$$

for all $i \in \mathbb{N} \cup\{0\}$. Setting $i=0$ and then $i=1$ shows that

$$
\left(a^{\ell(x)}, a^{\ell(y)}, a^{\ell(x y)}\right),\left(a^{\ell(x)}, a^{\ell(y)}, a^{\ell(x y)+p}\right) \in \Lambda(\circ, \phi),
$$

which implies that $a^{\ell(x y)} \phi=a^{\ell(x y)+p} \phi$, contradicting the injectivity of $\phi$.

Each case leads to a contradiction; this completes the proof.

The second technical result relates the lengths of representatives for an element and for powers of that element:

Lemma 20. Let $S$ be a semigroup admitting an injective unary automatic presentation $\left(a^{*}, \phi\right)$. For all $x \in S$ and $k \in \mathbb{N}$, one of the following conditions holds:

(1) $\ell\left(x^{k}\right) \leq n\left\lceil\log _{2} k\right\rceil$,

(2) $\left|\ell\left(x^{k}\right)-\ell(x)\right| \leq n\left\lceil\log _{2} k\right\rceil$,

where $n$ is the constant of Lemma 19.

Proof. Proceed by strong induction on $k$. For $k=1$, the values of $\left|\ell\left(x^{k}\right)-\ell(x)\right|$ and $\log _{2} k$ are both 0 , so condition (2) holds for $k=1$. 
For the induction step, suppose that $k>1$ and that for every $h<k$ one of the following conditions holds:

(1) $\ell\left(x^{h}\right) \leq n\left\lceil\log _{2} h\right\rceil$,

(2) $\left|\ell\left(x^{h}\right)-\ell(x)\right| \leq n\left\lceil\log _{2} h\right\rceil$.

The aim is to show that one of these two conditions holds for $h=k$. Now, $x^{k}=$ $x^{\lfloor k / 2\rfloor} x^{\lceil k / 2\rceil}$ and both $\lfloor k / 2\rfloor$ and $\lceil k / 2\rceil$ are strictly less than $k$ since $k>1$. Thus, by Lemma 19, one of the following holds:

$$
\begin{aligned}
\ell\left(x^{k}\right) & \leq n, \\
\left|\ell\left(x^{k}\right)-\ell\left(x^{\lfloor k / 2\rfloor}\right)\right| & \leq n, \\
\left|\ell\left(x^{k}\right)-l\left(x^{\lceil k / 2\rceil}\right)\right| & \leq n .
\end{aligned}
$$

Consider each case in turn:

(1) Suppose that $(23)$ holds: $\ell\left(x^{k}\right) \leq n$. Then $\left\lceil\log _{2} k\right\rceil \geq 1$ since $k \geq 2$, and so $\ell\left(x^{k}\right) \leq n\left\lceil\log _{2} k\right\rceil$. Thus condition (1) holds.

(2) Suppose that (24) holds: $\left|\ell\left(x^{k}\right)-\ell\left(x^{\lfloor k / 2\rfloor}\right)\right| \leq n$. By the induction hypothesis with $h=\lfloor k / 2\rfloor$, one of the following holds:

$$
\begin{aligned}
\ell\left(x^{\lfloor k / 2\rfloor}\right) & \leq n\left\lceil\log _{2}\lfloor k / 2\rfloor\right\rceil, \\
\left|\ell\left(x^{\lfloor k / 2\rfloor}\right)-\ell(x)\right| & \leq n\left\lceil\log _{2}\lfloor k / 2\rfloor\right\rceil .
\end{aligned}
$$

So there are two sub-cases:

(a) Suppose (26) holds. Then:

$$
\begin{array}{rlrl} 
& \ell\left(x^{k}\right) & \\
= & \left|\ell\left(x^{k}\right)-\ell\left(x^{\lfloor k / 2\rfloor}\right)+\ell\left(x^{\lfloor k / 2\rfloor}\right)\right| & & \\
\leq & \left|\ell\left(x^{k}\right)-\ell\left(x^{\lfloor k / 2\rfloor}\right)\right|+\ell\left(x^{\lfloor k / 2\rfloor}\right) & & \text { (by the triangle inequality) } \\
\leq & n+n\left\lceil\log _{2}\lfloor k / 2\rfloor\right\rceil & & \text { (by (24) and (26)) } \\
= & n\left\lceil\log _{2}\lfloor k / 2\rfloor+1\right\rceil & & \\
\leq & n\left\lceil\log _{2} k\right\rceil, &
\end{array}
$$

and so condition (1) holds.

(b) Suppose (27) holds. Then:

$$
\begin{array}{rlr} 
& \left|\ell\left(x^{k}\right)-\ell(x)\right| & \\
= & \left|\ell\left(x^{k}\right)-\ell\left(x^{\lfloor k / 2\rfloor}\right)+\ell\left(x^{\lfloor k / 2\rfloor}\right)-\ell(x)\right| & \\
\leq & \left|\ell\left(x^{k}\right)-\ell\left(x^{\lfloor k / 2\rfloor}\right)\right|+\left|\ell\left(x^{\lfloor k / 2\rfloor}\right)-\ell(x)\right| & \text { (by the triangle inequality) } \\
\leq & n+n\left\lceil\log _{2}\lfloor k / 2\rfloor\right\rceil & \\
= & n\left\lceil\log _{2}\lfloor k / 2\rfloor+1\right\rceil & \\
\leq & n\left\lceil\log _{2} k\right\rceil, &
\end{array}
$$

and so condition (2) holds. 
(3) Suppose that (25) holds, that $\left|\ell\left(x^{k}\right)-l\left(x^{\lceil k / 2\rceil}\right)\right| \leq n$. By the induction hypothesis with $h=\lceil k / 2\rceil$, one of the following holds

$$
\begin{aligned}
\ell\left(x^{\lceil k / 2\rceil}\right) & \leq n\left\lceil\log _{2}\lceil k / 2\rceil\right\rceil, \\
\left|\ell\left(x^{\lceil k / 2\rceil}\right)-\ell(x)\right| & \leq n\left\lceil\log _{2}\lceil k / 2\rceil\right\rceil .
\end{aligned}
$$

Again there are two sub-cases: if (28) holds, then reasoning parallel to sub-case (2)(a) above shows that

$$
\ell\left(x^{k}\right) \leq n\left\lceil\log _{2}\lceil k / 2\rceil+1\right\rceil .
$$

If (29) holds, then reasoning parallel to sub-case 2(b) above shows that

$$
\left|\ell\left(x^{k}\right)-\ell(x)\right| \leq n\left\lceil\log _{2}\lceil k / 2\rceil+1\right\rceil .
$$

If $k$ is even, then $\log _{2}\lceil k / 2\rceil+1=\log _{2} k$. If $k$ is odd, then $\log _{2}\lceil k / 2\rceil+1=$ $\log _{2}(k+1)$ and $\left\lceil\log _{2}(k+1)\right\rceil=\left\lceil\log _{2} k\right\rceil$, and so $\left\lceil\log _{2}\lceil k / 2\rceil+1\right\rceil=\left\lceil\log _{2} k\right\rceil$. Therefore, if (28) holds, then, regardless of whether $k$ is even or odd, it follows from (30) that

$$
\ell\left(x^{k}\right) \leq n\left\lceil\log _{2} k\right\rceil,
$$

and so condition (1) holds. On the other hand, if (29) holds, then similarly it follows from (31) that

$$
\left|\ell\left(x^{k}\right)-\ell(x)\right| \leq n\left\lceil\log _{2} k\right\rceil,
$$

and so condition (2) holds.

Theorem 21. Any unary FA-presentable semigroup satisfies a Burnside identity.

Proof. Let $S$ be a unary FA-presentable semigroup. By Theorem 9 , let $\left(a^{*}, \phi\right)$ be an injective unary automatic presentation for $S$.

Let $s \in S$. Then, by Lemma 20, for any $k \in \mathbb{N}$, one of the following holds:

$$
\begin{aligned}
\ell\left(s^{k}\right) & \leq n\left\lceil\log _{2} k\right\rceil \\
\left|\ell\left(s^{k}\right)-\ell(s)\right| & \leq n\left\lceil\log _{2} k\right\rceil,
\end{aligned}
$$

where $n$ is the constant of Lemma 19 .

Choose $h$ such that $h>3 n\left\lceil\log _{2} h\right\rceil$. Then for each $k<h$, there are only $3 n\left\lceil\log _{2} h\right\rceil$ possible values for $\ell\left(s^{k}\right)$, since $\ell\left(s^{k}\right)$ is either within $n\left\lceil\log _{2} h\right\rceil$ of $\ell(s)$ or at most $n\left\lceil\log _{2} h\right\rceil$. Since $h$ exceeds $3 n\left\lceil\log _{2} h\right\rceil$, by the pigeon-hole principle there exist $k_{s}$ and $k_{s}^{\prime}$, with $k_{s}<k_{s}^{\prime}<h$, such that $\ell\left(s^{k_{s}}\right)=\ell\left(s^{k_{s}^{\prime}}\right)$. Let $m_{s}=k_{s}^{\prime}-k_{s}$; then $0<m_{s}<h$ and $\ell\left(s^{k_{s}}\right)=\ell\left(s^{k_{s}+m_{s}}\right)$. So $s^{k_{s}}=s^{k_{s}+m_{s}}$, and it follows that the index and period of $s$ are less than $h$, which is dependent only on $(L, \phi)$. Let $k=\max \left\{k_{s}: s \in S\right\}$ and $m=\operatorname{lcm}\left\{m_{s}: s \in S\right\}$. Since there are only finitely many possibilities for $k_{s}$ and $m_{s}$, both $k$ and $m$ exist. Then $s^{k}=s^{k+m}$ for any element of $s$, and so $S$ satisfies the Burnside identity $x^{k}=x^{k+m}$. 
Margolis [Personal communication] posed the following question:

Question 22. Do all FA-presentable semigroups satisfy some non-trivial semigroup identity?

All known classes of FA-presentable semigroup satisfy some semigroup identity; see the various examples in [3]. Additionally, those semigroup constructions under which the class of FA-presentable semigroups is known to be closed [4] are also constructions under which the class of semigroups satisfying non-trivial identities is closed. Theorem 21 is further, albeit limited, evidence in favour of a positive answer to this question.

\section{Green's relations \& Schützenberger groups}

This section is devoted to describing the Green's relations $\mathcal{H}, \mathcal{R}, \mathcal{L}, \mathcal{D}$, and $\mathcal{J}$ for unary FA-presentable semigroups. The reader is assumed to be familiar with the definitions and basic theory of Green's relations; for background information, see [9, Ch. 2].

The following result is immediate:

Corollary 23. In a unary FA-presentable semigroup, Green's relations $\mathcal{D}$ and $\mathcal{J}$ coincide.

Proof. A unary FA-presentable semigroup is periodic by Theorem 21, and $\mathcal{D}=\mathcal{J}$ in periodic semgroups by [9, Proposition 2.1.4].

Since all the Green's relations are first-order definable equivalence relations, the following result is an immediate consequence of Theorem 8:

Proposition 24. In a unary FA-presentable semigroup, there are only finitely many infinite $\mathcal{J}-, \mathcal{D}_{-}, \mathcal{R}-, \mathcal{L}_{-}$, and $\mathcal{H}$-classes, and the finite ones are of bounded size.

The next result says, essentially, that the eggbox diagram for a $\mathcal{D}$-class (see $[9$, Section 2.2]) cannot have both infinitely many rows and infinitely many columns:

Proposition 25. In a unary FA-presentable semigroup, a $\mathcal{D}$-class cannot contain both infinitely many $\mathcal{R}$-classes and infinitely many $\mathcal{L}$-classes.

Proof. Suppose a unary FA-presentable semigroup $S$ has some $\mathcal{D}$-class $D$ that contains infinitely many $\mathcal{R}$-classes and infinitely many $\mathcal{L}$-classes. Then since there are infinitely many $\mathcal{L}$-classes in $D$ and every $\mathcal{H}$-class contains at least one element, every $\mathcal{R}$-class of $D$ is infinite. So there are infinitely many infinite $\mathcal{R}$-classes in $S$. Since $\mathcal{R}$ is an equivalence relation, this contradicts Theorem 8 . So no such $\mathcal{D}$-class can exist. 
In order to strengthen Proposition 24 to show that the $\mathcal{H}$-classes of a unary FApresentable semigroup are always finite, and in fact of bounded size, ideas from the theory of Schützenberger groups are required. The necessary definitions are recalled here; see [6, Section 2.4] for further background.

Definition 26. Let $S$ be a semigroup. Let $H$ be an $\mathcal{H}$-class of $S$ and let $h_{0}$ be an arbitrary element of $H$. The semigroup $S$ acts by right multiplication on the set of $\mathcal{H}$-classes in the $\mathcal{R}$-class containing $H$ with a sink adjoined. The right stabilizer of $H$ is denoted $\operatorname{Stab}(H)$ :

$$
\operatorname{Stab}(H)=\{s \in S: H s=H\}=\left\{s \in S: h_{0} s \mathcal{H} h_{0}\right\} .
$$

Define a relation $\sigma(H)$ on $\operatorname{Stab}(H)$ by

$$
(s, t) \in \sigma(H) \Longleftrightarrow(\forall h \in H)(h s=h t) .
$$

This relation is a congruence, and its definition is equivalent to

$$
(s, t) \in \sigma(H) \Longleftrightarrow\left(h_{0} s=h_{0} t\right) .
$$

The factor semigroup $\Gamma(H)=\operatorname{Stab}(H) / \sigma(H)$ is actually a group, called the Schützenberger group of $H$. The group $\Gamma(H)$ acts regularly on $H$; thus $|H|=|\Gamma(H)|$, and if $H$ is a group then $H \simeq \Gamma(H)$.

Proposition 27. Any unary FA-presentable semigroup has a bound on the size of its $\mathcal{H}$-classes.

Proof. Let $(L, \phi)$ be a unary automatic presentation for $S$. Choose $w \in L$. Let $h_{0}=w \phi$; the aim is to show that $H_{h_{0}}$ is finite.

The set $\operatorname{Stab}\left(H_{h_{0}}\right)$ is first-order definable by (34); thus the set of words $K=\left\{w \in L: w \phi \in \operatorname{Stab}\left(H_{h_{0}}\right)\right\}$ is regular. Thus $\left(K,\left.\phi\right|_{K}\right)$ is a unary automatic presentation for the subsemigroup $\operatorname{Stab}\left(H_{h_{0}}\right)$.

The congruence $\sigma\left(H_{h_{0}}\right)$ is first-order definable by (36). Thus the Schützenberger group $\Gamma\left(H_{h_{0}}\right)=\operatorname{Stab}\left(H_{h_{0}}\right) / \sigma\left(H_{h_{0}}\right)$ admits a unary automatic presentation $\left(K,\left.\phi\right|_{K} \sigma^{\#}\right)$, where $\sigma^{\#}$ is the natural map from $\operatorname{Stab}\left(H_{h_{0}}\right)$ to $\operatorname{Stab}\left(H_{h_{0}}\right) / \sigma\left(H_{h_{0}}\right)$. Thus, by Proposition 10, the group $\Gamma\left(H_{h_{0}}\right)$ is finite.

Since $w \in L$ (and thus $h_{0} \in S$ ) was arbitrary, every Schützenberger group of an $\mathcal{H}$-class of $S$ is finite. Thus every $\mathcal{H}$-class of $S$ is finite. Since $\mathcal{H}$ is an equivalence relation on $S$, there is a bound on the size of the $\mathcal{H}$-classes of $S$ by Theorem 8 .

Proposition 28. The principal factor arising from any $\mathcal{J}$-class of a unary FApresentable semigroup is either completely 0-simple or a null semigroup.

Proof. Let $T$ be some principal factor of a unary FA-presentable semigroup $S$. By [9, Theorem 3.1.6(2)], $T$ is either 0-simple or null. If it is null, there is nothing more to prove. So suppose $T$ is 0 -simple. Since $S$ is periodic by Theorem 21, so is $T$. In particular, $T$ is group-bound. Thus, by [9, Theorem 3.2.11], $T$ is completely 0-simple. 
The following example shows that there do exist unary FA-presentable semigroups with an arbitrary finite number of infinite $\mathcal{D}$-classes and an infinite number of finite ones.

Example 29. Let $S$ be a countable right zero semigroup, which is unary FA-presentable by Example 11.

Let $T$ be the countable chain $\left\{t_{0}, t_{1}, \ldots\right\}$ with ordering $t_{i} \leq t_{j}$ if and only if $i \leq j$. Let $\psi: a^{*} \rightarrow T$ be defined by $a^{n} \psi=t_{n}$. Then

$$
\Lambda(=, \psi)=\left\{\left(a^{p}, a^{p}\right): p \in \mathbb{N} \cup\{0\}\right\}
$$

and

$$
\begin{aligned}
\Lambda(\circ, \psi)= & \left\{\left(a^{p}, a^{q}, a^{r}\right): a^{p} \psi \circ a^{q} \psi=a^{r} \psi p, q, r \in \mathbb{N} \cup\{0\}\right\} \\
= & \left\{\left(a^{p}, a^{q}, a^{r}\right): t_{p} \circ t_{q}=t_{r}, p, q, r \in \mathbb{N} \cup\{0\}\right\} \\
= & \left\{\left(a^{p}, a^{q}, a^{r}\right): t_{q}=t_{r}, t_{q} \leq t_{p}, p, q, r \in \mathbb{N} \cup\{0\}\right\} \\
& \cup\left\{\left(a^{p}, a^{q}, a^{r}\right): t_{p}=t_{r}, t_{p} \leq t_{q}, p, q, r \in \mathbb{N} \cup\{0\}\right\} \\
= & \left\{\left(a^{p}, a^{q}, a^{q}\right): p, q \in \mathbb{N} \cup\{0\}, q \leq p\right\} \\
& \cup\left\{\left(a^{p}, a^{q}, a^{p}\right): p, q \in \mathbb{N} \cup\{0\}, p \leq q\right\}
\end{aligned}
$$

and so $\Lambda(=, \psi)$ and $\Lambda(\circ, \psi)$ are regular. Thus $\left(a^{*}, \psi\right)$ is a unary automatic presentation for $T$.

Let $k \in \mathbb{N} \cup\{0\}$. Let $U_{0}=T$. For each $i=1, \ldots, k$, let $S_{i}$ be a copy of $S$ and let $U_{i}$ be the ordinal sum of $S_{i}$ and $U_{i-1}$ with respect to the ordering $S>U_{i}$. (See Section 9.1 for the definition of ordinal sums.) Then by iterated applicaton of Proposition 30, $U_{k}$ is unary FA-presentable.

Now, in $U_{k}$, products in each subsemigroup $U_{i}$ are as before, and if $x \in U_{i}$ and $y \in U_{j}$ with $i<j$, then $x y=y x=x$. So in $U_{k}$, the $\mathcal{R}$-class, and thus the $\mathcal{D}$-class of any element of $S_{i}$ is the whole of $S_{i}$, and the $\mathcal{D}$-class of any element $t \in T$ is the singleton set $\{t\}$. So $U_{k}$ contains countably many finite (singleton) $\mathcal{D}$-classes inside $T$, and $k$ countable $\mathcal{D}$-classes, namely the $S_{i}$.

Although the results in this section describe the possible $\mathcal{J}_{-}, \mathcal{D}-, \mathcal{R}_{-}, \mathcal{L}-$, and $\mathcal{H}_{-}$ classes and principal factors of a unary FA-presentable semigroup, what is lacking is a description of how these interact. In particular, no characterization is yet known of unary FA-presentable semilattices (where all Green's relations are simply the equality relation). This seems to be the major obstacle on the way to a complete characterization of unary FA-presentable semigroups.

\section{Constructions}

This section examines the interaction of the class of unary FA-presentable semigroups and four semigroup constructions: extensions and subsemigroups, Rees matrix semigroups, direct products, and free products. 


\subsection{Extensions and subsemigroups}

The ordinal sum of two semigroup $S$ and $T$ with respect to the ordering $S>T$, is the disjoint union of $S$ and $T$ with the multiplication of two elements of $S$ or two elements of $T$ as before and the product of $s \in S$ and $t \in T$ defined to be $t$ : that is, $s t=t s=t$ for all $s \in S$ and $t \in T$. So this ordinal sum is a particular ideal extension of $T$ by $S$. (The notion of an ordinal sum is due to Clifford [5], who defined it for an arbitrary collection of semigroups indexed by a totally ordered semigroup, and with each semigroup admitting a particular type of total order.)

Proposition 30. The ordinal sum of two unary FA-presentable semigroups is itself unary FA-presentable.

Proof. Let $S$ and $T$ be semigroups admitting unary automatic presentations $(K, \phi)$ (where $K \subseteq a^{*}$ ) and $(L, \psi)$ (where $L \subseteq b^{*}$ ) respectively. (Note that Theorem 9 cannot be applied here because one or both of $S$ and $T$ may be finite.) Let $U$ be the ordinal sum of $S$ and $T$ with respect to the ordering $S>T$.

Define the following homomorphisms:

$$
\begin{array}{ll}
\eta: a^{*} \rightarrow c^{*}, & a \mapsto c^{2}, \\
\vartheta: b^{*} \rightarrow c^{*}, & b \mapsto c^{2} .
\end{array}
$$

Since regularity is preserved under homomorphism, $K^{\prime}=K \eta$ and $L^{\prime}=L \vartheta$ are regular. Notice that $K^{\prime}, L^{\prime} \subseteq\left\{c^{2}\right\}^{*}$, so $K^{\prime}$ and $c L^{\prime}$ are disjoint. Let $M=K^{\prime} \cup c L^{\prime}$. Now define a map

$$
\chi: M \rightarrow U, \quad\left\{\begin{array}{l}
c^{2 k} \mapsto a^{k} \phi \\
c^{2 k+1} \mapsto b^{k} \psi
\end{array}\right.
$$

By the definition of $M$, this map is well-defined.

Let $\mathfrak{A}$ recognize conv $(\Lambda(\circ, \phi))$ and $\mathfrak{B}$ recognize $\operatorname{conv}(\Lambda(\circ, \psi))$. In $\mathfrak{A}$, each edge is labelled by a triple whose components are either $a$ or $\$$. On every edge, replace each component $a$ with $c^{2}$ and each component $\$$ with $\$^{2}$. Call the resulting automaton $\mathfrak{A}^{\prime}$. Similarly, on every edge of $\mathfrak{B}$, replace each component $b$ with $c^{2}$ and each component $\$$ with $\$^{2}$ to obtain an automaton $\mathfrak{B}^{\prime}$. It is easy to see that

$$
\operatorname{conv}\left(\Lambda\left(\circ,\left.\chi\right|_{S \chi^{-1}}\right)\right)=L\left(\mathfrak{A}^{\prime}\right) \text { and } \operatorname{conv}\left(\Lambda\left(\circ,\left.\chi\right|_{T \chi^{-1}}\right)\right)=(c, c, c) L\left(\mathfrak{B}^{\prime}\right) .
$$

So $\Lambda\left(\circ,\left.\chi\right|_{S \chi^{-1}}\right)$ and $\Lambda\left(\circ,\left.\chi\right|_{T \chi^{-1}}\right)$ are both regular. Now,

$$
\begin{aligned}
& \Lambda(\circ, \chi) \\
= & \left\{(u, v, w): u, v, w \in c^{*}:(u \chi) \circ(v \chi)=w \chi\right\} \\
= & \Lambda\left(\circ,\left.\chi\right|_{S \chi^{-1}}\right) \cup \Lambda\left(\circ,\left.\chi\right|_{T \chi^{-1}}\right) \\
& \quad \cup\left\{\left(c^{2 k}, c^{2 m+1}, c^{2 m+1}\right),\left(c^{2 m+1}, c^{2 k}, c^{2 m+1}\right): k, m \in \mathbb{N} \cup\{0\}\right\},
\end{aligned}
$$

so $\Lambda(\circ, \chi)$ is regular. Thus $\left(c^{*}, \chi\right)$ is a unary automatic presentation for $U$. 
Recall that a subsemigroup $T$ of a semigroup $S$ has finite Rees index if the set $S-T$ is finite.

Proposition 31. The class of unary FA-presentable semigroups is closed under passing to subsemigroups of finite Rees index.

Proof. Let $S$ be a unary FA-presentable semigroup and let $T$ be a subsemigroup of $S$ of finite Rees index. Let $\left(a^{*}, \phi\right)$ be an injective unary automatic presentation for $S$. Let $K=(S-T) \phi^{-1}$. Since $S-T$ is finite and $\phi$ is injective, $K$ is a finite subset of $a^{*}$ and therefore regular. So $L=a^{*}-K$ is regular, and $\left.L \phi\right|_{L}=T$. Finally,

$$
\begin{aligned}
\Lambda\left(=,\left.\phi\right|_{L}\right) & =\Lambda(=, \phi) \cap(L \times L), \\
\Lambda\left(\circ,\left.\phi\right|_{L}\right) & =\Lambda(\circ, \phi) \cap(L \times L \times L),
\end{aligned}
$$

and so $\left(L,\left.\phi\right|_{L}\right)$ is a unary automatic presentation for $T$.

Corollary 32. Let $S$ be a semigroup. Then $S$ is unary FA-presentable if and only if $S^{0}$ is unary FA-presentable.

Proof. For any semigroup $S$, the semigroup $S^{0}$ is the ordinal sum of $S$ and the trivial semigroup $\{0\}$ with respect to the ordering $S>\{0\}$. Thus, by Proposition 30, $S^{0}$ is unary FA-presentable if $S$ is. In the other direction, $S$ is a finite Rees index subsemigroup of $S^{0}$ and so $S$ is unary FA-presentable if $S^{0}$ is by Proposition 31 .

[Proposition 12 could also be deduced from Proposition 31 and Proposition 30 (since $S^{1}$ is the ordinal sum of the semigroup $S$ and trivial semigroup $\{1\}$ with respect to the ordering $\{1\}>S$ ) in a manner similar to Corollary 32.]

The converse of Proposition 31 does not hold: the following example gives an example of a semigroup $S$ with a subsemigroup $T$ of finite Rees index (indeed, $|S-T|=1$ ) with $T$ admitting a unary automatic presentation and $S$ not admitting any automatic presentation, unary or otherwise.

Example 33. Define a semilattice $S$ as follows. The set of elements is $\left\{s_{i}, t_{i}: i \in\right.$ $\mathbb{N} \cup\{0\}\}$, and the order $\leq$ is defined on $S$ as follows: for all $i, j \in \mathbb{N}$,

$$
\begin{aligned}
t_{i} \leq t_{j} & \Longleftrightarrow i \leq j \\
t_{i} \leq s_{j} & \Longleftrightarrow i \leq j \\
s_{i} \leq s_{j} & \Longleftrightarrow i=j \\
s_{i} \not \leq t_{j} . &
\end{aligned}
$$

The Hasse diagram for $(S, \leq)$ is as illustrated in Fig. 1.

Let $Y \subseteq \mathbb{N} \cup\{0\}$ be non-recursively enumerable. Let $U=S \cup\{e\}$ and extend the relation $\leq$ to $S$ as follows: for $i \in \mathbb{N}$, by defining

$$
\begin{aligned}
t_{i} & \leq e \\
s_{i} & \leq e \Longleftrightarrow i \in Y .
\end{aligned}
$$




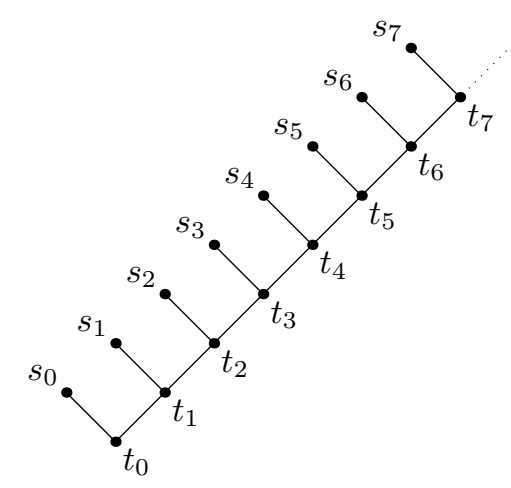

Fig. 1. Hasse diagram for $(S, \leq)$.

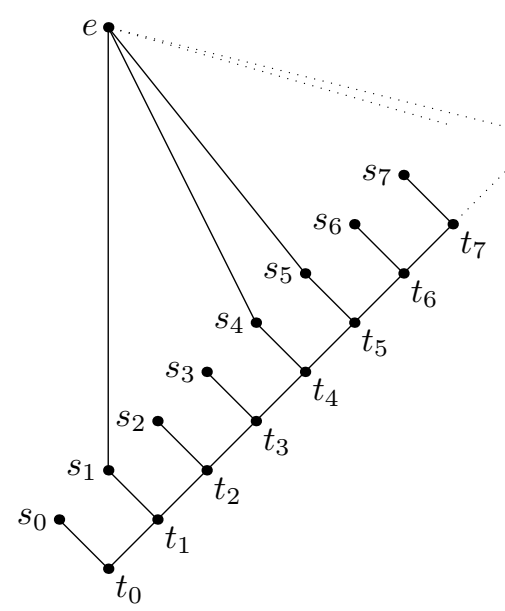

Fig. 2. Hasse diagram for $(U, \leq)$, assuming for the sake of illustration that $1,4,5$ lie in $Y$.

The Hasse diagram for $(S, \leq)$ is as illustrated in Fig. 2 .

Define a mapping

$$
\phi: a^{*} \rightarrow S, \quad\left\{\begin{array}{l}
a^{2 i} \mapsto s_{i} \\
a^{2 i+1} \mapsto t_{i} .
\end{array}\right.
$$

First, notice that $\phi$ is injective, so $\Lambda(=, \phi)=\left\{a^{n}, a^{n}: n \in \mathbb{N} \cup\{0\}\right\}$. Furthermore,

$$
\begin{aligned}
& \Lambda(\leq, \phi) \\
= & \left\{\left(a^{m}, a^{n}\right): m, n \in \mathbb{N} \cup\{0\}, a^{m} \phi \leq a^{n} \phi\right\}
\end{aligned}
$$




$$
\begin{gathered}
=\left\{\left(a^{2 i}, a^{2 j}\right): i, j \in \mathbb{N} \cup\{0\}, a^{2 i} \phi \leq a^{2 j} \phi\right\} \\
\cup\left\{\left(a^{2 i}, a^{2 j+1}\right): i, j \in \mathbb{N} \cup\{0\}, a^{2 i} \phi \leq a^{2 j+1} \phi\right\} \\
\cup\left\{\left(a^{2 i+1}, a^{2 j+1}\right): i, j \in \mathbb{N} \cup\{0\}, a^{2 i+1} \phi \leq a^{2 j+1} \phi\right\} \\
\cup\left\{\left(a^{2 i+1}, a^{2 j}\right): i, j \in \mathbb{N} \cup\{0\}, a^{2 i+1} \phi \leq a^{2 j} \phi\right\} \\
=\left\{\left(a^{2 i}, a^{2 j}\right): i, j \in \mathbb{N} \cup\{0\}, s_{i} \leq s_{j}\right\} \\
\cup\left\{\left(a^{2 i}, a^{2 j+1}\right): i, j \in \mathbb{N} \cup\{0\}, s_{i} \leq t_{j}\right\} \\
\cup\left\{\left(a^{2 i+1}, a^{2 j+1}\right): i, j \in \mathbb{N} \cup\{0\}, t_{i} \leq t_{j}\right\} \\
\cup\left\{\left(a^{2 i+1}, a^{2 j}\right): i, j \in \mathbb{N} \cup\{0\}, t_{i} \leq s_{j}\right\} \\
=\left\{\left(a^{2 i}, a^{2 j}\right): i, j \in \mathbb{N} \cup\{0\}, i=j\right\} \\
\cup \emptyset \quad\left(\text { since } s_{i} \leq t_{j}\right) \\
\cup\left\{\left(a^{2 i+1}, a^{2 j+1}\right): i, j \in \mathbb{N} \cup\{0\}, i \leq j\right\} \\
\cup\left\{\left(a^{2 i+1}, a^{2 j}\right): i, j \in \mathbb{N} \cup\{0\}, i \leq j\right\},
\end{gathered}
$$

which is regular. Thus $\left(a^{*}, \phi\right)$ is a unary automatic presentation for $(S, \leq)$.

Suppose for reductio ad absurdum that $(U, \leq)$ admits an automatic presentation $(K, \phi)$. The aim is obtain a contradiction by showing that the set $Y$ is effectively enumerable. Without loss of generality, assume by Proposition 5 that $\phi$ is injective. Let

$$
\sigma(x, y)=(x<y) \wedge(\forall z \in U)(x<z \Longrightarrow y \leq z) .
$$

For any $x \in U$, let $\Sigma(x)$ be the set of elements $y \in U$ such that $\sigma(x, y)$. Then $\Sigma(x)$ consists of the set of minimal elements lying above $x$ in the semilattice. That is,

$$
\begin{aligned}
& \Sigma\left(s_{i}\right)= \begin{cases}\{e\} & \text { if } i \in Y \\
\emptyset & \text { if } i \notin Y\end{cases} \\
& \Sigma\left(t_{i}\right)=\left\{t_{i+1}, s_{i}\right\} \\
& \Sigma(e)=\emptyset .
\end{aligned}
$$

Since $\sigma$ is a first-order formula, given a word representing some element $x$, a set of at most two words representing the elements of the set $\Sigma(x)$ can be found effectively.

First, let $u_{0} \in K$ and $v \in K$ be the unique words with $u_{0} \phi=t_{0}$ and $v \phi=e$. The procedure enumerating $Y$ stores a word $u_{i}$ and the subscript $i$ between iterations.

Each iteration of the procedure is as follows: For a word $u_{i}$ representing $t_{i}$, find the set of words representing $\Sigma\left(t_{i}\right)$. This set consists of two words $w_{1}, w_{2}$, one representing $t_{i+1}$ and one representing $s_{i}$. Find words representing the elements of the sets $\Sigma\left(w_{1} \phi\right)$ and $\Sigma\left(w_{2} \phi\right)$; whichever word $w_{j}$ has $\Sigma\left(w_{j} \phi\right)$ consisting of exactly two words must represent $t_{i+1}$. Set $u_{i+1}=w_{j}$. The other word represents $s_{i}$ and so the set of words representing $\Sigma\left(s_{i}\right)$ can be effectively calculated. This set is nonempty if and only if $i \in Y$ : in this case, output the subscript $i$. This completes the iteration and the procedure continues from the start of this paragraph. 


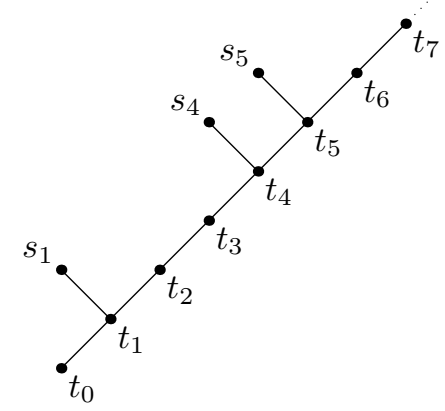

Fig. 3. Hasse diagram for $(T, \leq)$, assuming for the sake of illustration that $1,4,5$ lie in $Y$.

This procedure enumerates the elements of $Y$. This is a contradiction since $Y$ is not recursively enumerable, and so $(U, \leq)$ cannot admit an automatic presentation.

The following example shows that Proposition 31 does not generalize to arbitrary subsemigroups.

Example 34. Let $(S, \leq)$ be the semilattice from Example 33. Let $Y \subseteq \mathbb{N} \cup\{0\}$ be non-recursively enumerable and let $T=\left\{t_{i}: i \in \mathbb{N} \cup\{0\}\right\} \cup\left\{s_{i}: i \in Y\right\}$. Then $T$ is a subsemilattice of $S$, and the Hasse diagram of $(T, \leq)$ is as illustrated in Fig. 3.

Suppose for reductio ad absurdum that $(T, \leq)$ admits an automatic presentation $(K, \phi)$. The aim is obtain a contradiction by showing that the set $Y$ is effectively enumerable. Without loss of generality, assume that $\phi$ is injective. Let

$$
\sigma(x, y)=(x<y) \wedge(\forall z \in U)(x<z \Longrightarrow y \leq z) .
$$

For any $x \in U$, let $\Sigma(x)$ be the set of elements $y \in U$ such that $\sigma(x, y)$. Then $\Sigma(x)$ consists of the set of minimal elements lying above $x$ in the semilattice. That is

$$
\begin{aligned}
& \Sigma\left(s_{i}\right)=\emptyset \\
& \Sigma\left(t_{i}\right)= \begin{cases}\left\{t_{i+1}, s_{i}\right\} & \text { if } i \in Y . \\
\left\{t_{i+1}\right\} & \text { if } i \notin Y\end{cases}
\end{aligned}
$$

Since $\sigma$ is a first-order formula, given a word representing some element $x$, a set of at most two words representing the elements of the set $\Sigma(x)$ can be found effectively.

First, let $u_{0} \in K$ be the unique word with $u_{0} \phi=t_{0}$. The procedure enumerating $Y$ stores a word $u_{i}$ and the subscript $i$ between iterations.

Each iteration of the procedure is as follows: For a word $u_{i}$ representing $t_{i}$, find the set of words representing $\Sigma\left(t_{i}\right)$. If this set consists of a single word $w$, set $u_{i+1}=w$ and continue from the start of this paragraph. If the set consists of two words $w_{1}, w_{2}$, then one of these words represents $t_{i+1}$ and one represents $s_{i}$. Find words representing the elements of the sets $\Sigma\left(w_{1} \phi\right)$ and $\Sigma\left(w_{2} \phi\right)$; whichever word $w_{j}$ has $\Sigma\left(w_{j} \phi\right)$ non-empty must represent $t_{i+1}$. Set $u_{i+1}=w_{j}$. Output the index $i$, 
since in this case $i \in Y$. This completes the iteration and the procedure continues from the start of this paragraph.

This procedure enumerates the elements of $Y$. This is a contradiction since $Y$ is not recursively enumerable, and so $(T, \leq)$ cannot admit an automatic presentation.

\subsection{Rees matrix semigroups}

The next two results show, respectively, that the class of unary FA-presentable semigroups is closed under forming finite-by-finite Rees matrix semigroups, and that it includes all finite-by-countable Rees matrix semigroups over finite semigroups. Recall that a Rees matrix semigroup $\mathcal{M}[T ; I, J ; P]$, where $T$ is a semigroup, $I$ and $J$ are abstract (possibly infinite) index sets, and $P$ is a $J \times I$ matrix with entries from $T$, is a semigroup with underlying set $I \times T \times J$ and multiplication given by

$$
(i, t, j)(k, u, \ell)=\left(i, t p_{j, k} u, \ell\right) .
$$

(See [6, Section 3.1] or [9, Section 3.2] for further information on Rees matrix semigroups.)

Proposition 35. Any finite-by-finite Rees matrix semigroup over a unary FA-presentable semigroup is unary FA-presentable. More precisely, if $S=\mathcal{M}[T ; I, J ; P]$, where $I$ and $J$ are finite, $T$ is unary FA-presentable, and $P$ is a $J \times I$ matrix over $T$, then $S$ is unary FA-presentable.

Proof. If $T$ is finite, so is $S$ and so $S$ is unary FA-presentable. So assume $T$ is infinite. Then by Theorem $9, T$ admits a unary automatic presentation $\left(a^{*}, \phi\right)$. Suppose that $I=\left\{0, \ldots, n_{i}-1\right\}$ and $J=\left\{0, \ldots, n_{j}-1\right\}$.

Let $k=n_{i} n_{j}$. Define a map

$$
\psi: b^{*} \rightarrow S, \quad b^{\alpha} \psi=\left((\alpha \bmod k) \bmod n_{j}, a^{\lfloor\alpha / k\rfloor} \phi,\left\lfloor(\alpha \bmod k) / n_{j}\right\rfloor\right),
$$

where $\alpha \bmod k$ is interpreted as the unique $h \in \mathbb{N}$ with $0 \leq h<k$ and $h \equiv \alpha$ $(\bmod k)$. Since $n_{j} \mid k$,

$$
b^{\alpha} \psi=\left(\alpha \bmod n_{j}, a^{\lfloor\alpha / k\rfloor} \phi,\left\lfloor(\alpha \bmod k) / n_{j}\right\rfloor\right) .
$$

The idea of the map $\psi$ is that $b^{m k}, b^{m k+1}, \ldots, b^{m k+(k-1)}$ represent all elements of $S$ of the form $\left(i, a^{m} \phi, j\right)$, with the exponent taken modulo $k$ determining $i$ and $j$.

For all $i \in I, j \in J$, let $p_{j, i} \in G$ be the $(j, i)$-th element of $P$. The relation

$$
R_{j, i}^{\prime}=\left\{\left(a^{\beta_{1}}, a^{\beta_{2}}, a^{\beta_{3}}\right): \beta_{i} \in \mathbb{N} \cup\{0\},\left(a^{\beta_{1}} \phi\right) p_{j, i}\left(a^{\beta_{2}} \phi\right)=a^{\beta_{3}} \phi\right\}
$$

is first-order definable in terms of $\phi$ and so is regular. From an automaton recognizing $\operatorname{conv} R_{j, i}^{\prime}$ it is easy to construct one recognizing $\operatorname{conv} R_{j, i}$, where

$$
\begin{aligned}
R_{j, i} & =\left\{\left(b^{k \beta_{1}+\beta_{1}^{\prime}}, b^{k \beta_{2}+\beta_{2}^{\prime}}, b^{k \beta_{3}+\beta_{3}^{\prime}}\right): \beta_{i} \in \mathbb{N} \cup\{0\}, \beta_{i}^{\prime}<k,\left(a^{\beta_{1}} \phi\right) p_{j, i}\left(a^{\beta_{2}} \phi\right)=a^{\beta_{3}} \phi\right\} \\
& =\left\{\left(b^{\alpha_{1}}, b^{\alpha_{2}}, b^{\alpha_{3}}\right): \alpha_{i} \in \mathbb{N} \cup\{0\},\left(a^{\left\lfloor\alpha_{1} / k\right\rfloor} \phi\right) p_{j, i}\left(a^{\left\lfloor\alpha_{2} / k\right\rfloor} \phi\right)=a^{\left\lfloor\alpha_{3} / k\right\rfloor} \phi\right\} .
\end{aligned}
$$


Then

$$
\begin{aligned}
& \Lambda(\circ, \psi) \\
&=\left\{\left(b^{\alpha}, b^{\beta}, b^{\gamma}\right): \alpha, \beta, \gamma \in \mathbb{N} \cup\{0\},\left(b^{\alpha} \psi\right)\left(b^{\beta} \psi\right)=\left(b^{\gamma} \psi\right)\right\} \\
&=\left\{\left(b^{\alpha}, b^{\beta}, b^{\gamma}\right): \alpha, \beta, \gamma \in \mathbb{N} \cup\{0\},\right. \\
&\left(\alpha \bmod n_{j}, a^{\lfloor\alpha / k\rfloor} \phi,\left\lfloor(\alpha \bmod k) / n_{j}\right\rfloor\right) \\
&\left(\beta \bmod n_{j}, a^{\lfloor\beta / k\rfloor} \phi,\left\lfloor(\beta \bmod k) / n_{j}\right\rfloor\right) \\
&=\left.\left(\gamma \bmod n_{j}, a^{\lfloor\gamma / k\rfloor} \phi,\left\lfloor(\gamma \bmod k) / n_{j}\right\rfloor\right)\right\} \\
&=\left\{\left(b^{\alpha}, b^{\beta}, b^{\gamma}\right): \alpha, \beta, \gamma \in \mathbb{N} \cup\{0\},\right. \\
&\left(\alpha \bmod n_{j},\left(a^{\lfloor\alpha / k\rfloor} \phi\right) p_{\left\lfloor(\alpha \bmod k) / n_{j}\right\rfloor, \beta \bmod n_{j}}\left(a^{\lfloor\beta / k\rfloor} \phi\right),\left\lfloor(\beta \bmod k) / n_{j}\right\rfloor\right) \\
&\left.=\left(\gamma \bmod n_{j}, a^{\lfloor\gamma / k\rfloor} \phi,\left\lfloor(\gamma \bmod k) / n_{j}\right\rfloor\right)\right\} \\
&=\left\{\left(b^{\alpha}, b^{\beta}, b^{\gamma}\right): \alpha, \beta, \gamma \in \mathbb{N} \cup\{0\},\right. \\
& \alpha \bmod n_{j}=\gamma \bmod n_{j} \wedge\left\lfloor(\beta \bmod k) / n_{j}\right\rfloor=\left\lfloor(\gamma \bmod k) / n_{j}\right\rfloor \\
&\left.\wedge\left(a^{\lfloor\alpha / k\rfloor} \phi\right) p_{\left\lfloor(\alpha \bmod k) / n_{j}\right\rfloor, \beta \bmod n_{j}}\left(a^{\lfloor\beta / k\rfloor} \phi\right)=a^{\lfloor\gamma / k\rfloor} \phi\right\} \\
&=\left\{\left(b^{\alpha}, b^{\beta}, b^{\gamma}\right): \alpha, \beta, \gamma \in \mathbb{N} \cup\{0\},\right. \\
& \alpha \bmod n_{j}=\gamma \bmod n_{j} \wedge\left\lfloor(\beta \bmod k) / n_{j}\right\rfloor=\left\lfloor(\gamma \bmod k) / n_{j}\right\rfloor \\
&\left.\wedge\left(a^{\lfloor\alpha / k\rfloor}, a^{\lfloor\beta / k\rfloor}, a^{\lfloor\gamma / k\rfloor}\right) \in R_{\left.\left\lfloor(\alpha \bmod k) / n_{j}\right\rfloor, \beta \bmod n_{j}\right\}}\right\} \\
&=
\end{aligned}
$$

Since the relations $R_{j, i}$ are all regular, and since a finite automaton can track integers modulo $n_{j}$ and modulo $k$, it follows that $\Lambda(\circ, \psi)$ is regular, and hence $\left(b^{*}, \psi\right)$ is an automatic presentation for $S$.

The following example, which is a modified version of a discussion in [4, Section 8], shows that the converse of Proposition 35 does not hold:

Example 36. Let $F$ be the free semigroup with basis $\{x\}$. Form the Rees matrix semigroup $S=\mathcal{M}\left[F^{0} ; I, J ; P\right]$, where $I=J=\{1\}$ and let $P$ is the $J \times I$ matrix whose single entry is 0 . So the underlying set of $S$ is $\{1\} \times\left(\{0\} \cup\left\{x^{\alpha}: \alpha \in \mathbb{N}\right\}\right) \times\{1\}$, and every product in $T$ is $(1,0,1)$ because the single entry of $P$ is 0 .

Define a map

$$
\phi: a^{*} \rightarrow S, \quad a^{\alpha} \mapsto \begin{cases}(1,0,1) & \text { if } \alpha=0 \\ \left(1, x^{\alpha}, 1\right) & \text { if } \alpha \neq 0\end{cases}
$$


Then $\phi$ is injective, so $\Lambda(=, \phi)=\left\{\left(a^{\alpha}, a^{\alpha}\right): \alpha \in \mathbb{N} \cup\{0\}\right\}$, which is regular. Furthermore,

$$
\begin{aligned}
\Lambda(\circ, \phi) & =\left\{\left(a^{\alpha}, a^{\beta}, a^{\gamma}\right): \alpha, \beta, \gamma \in \mathbb{N} \cup\{0\},\left(a^{\alpha} \phi\right)\left(a^{\beta} \phi\right)=a^{\gamma} \phi\right\} \\
& =\left\{\left(a^{\alpha}, a^{\beta}, a^{\gamma}\right): \alpha, \beta, \gamma \in \mathbb{N} \cup\{0\}, a^{\gamma} \phi=(1,0,1)\right\} \\
& =\left\{\left(a^{\alpha}, a^{\beta}, \varepsilon\right): \alpha, \beta \in \mathbb{N} \cup\{0\}\right\},
\end{aligned}
$$

so that $\Lambda(\circ, \phi)$ is regular. Hence $\left(a^{*}, \phi\right)$ is a unary automatic presentation for $S$.

However, the base semigroup $F^{0}$ is finitely generated and infinite, and therefore cannot be unary FA-presentable by Corollary 14 .

Proposition 37. Any finite-by-countable Rees matrix semigroup over a finite semigroup is unary FA-presentable. More precisely, if $S=\mathcal{M}[T, I, J, P]$, where one of $I$ and $J$ is finite and the other countable, $T$ is finite, and $P$ is a $J \times I$ matrix over $T$, then $S$ is unary FA-presentable.

Proof. Let $S=\mathcal{M}[T, I, J, P]$. Assume that $I$ is finite and $J$ is countable, with $I=\left\{0, \ldots, n_{i}-1\right\}$ and $J=\mathbb{N} \cup\{0\}$. There are only finitely many distinct rows of the $J \times I$ matrix $P$. So some rows will appear only finitely many times, some will appear infinitely many times. Permute the rows as follows. The $p$ rows that appear only finitely many times are placed first, in rows 0 up to $p-1$. The $q$ rows that appear infinitely many times are arranged periodically from $p$ onwards, so that for any $j \geq p$, row $j$ is identical to row $((j-p) \bmod q)+p$. Permuting the rows thus yields a semigroup isomorphic to the original Rees matrix semigroup, so assume without loss of generality that $P$ has already been arranged in this way.

Let the elements of the finite semigroup $T$ be $t_{0}, \ldots, t_{r-1}$. Let $k=n_{i} r$. Define a map

$$
\phi: a^{*} \rightarrow S, \quad a^{\alpha} \mapsto\left(\lfloor(\alpha \bmod k) / r\rfloor, t_{\alpha \bmod r},\lfloor\alpha / k\rfloor\right)
$$

It is easy to see that $\phi$ is injective and so $\Lambda(=, \phi)=\left\{\left(a^{\alpha}, a^{\alpha}\right): \alpha \in \mathbb{N} \cup\{0\}\right\}$, which is regular.

For all $i \in I, j \in \mathbb{N}$, let $p_{j, i} \in T$ be the $(j, i)$-th element of $P$. The relation

$$
R_{j, i}=\left\{\left(a^{\alpha}, a^{\beta}, a^{\gamma}\right): \alpha, \beta, \gamma \in \mathbb{N} \cup\{0\}, t_{\alpha \bmod r} p_{j, i} t_{\beta \bmod r}=t_{\gamma \bmod r}\right\}
$$

is regular since a finite automaton can track the $\alpha, \beta$, and $\gamma$ modulo $r$. Notice further that for any $i \in I, j \in \mathbb{N}$ with $j \geq p$, the relations $R_{j, i}$ and $R_{((j-p) \bmod q)+p, i}$ are equal. For convenience later in the proof, define

$$
\pi: \mathbb{N} \rightarrow \mathbb{N}, \quad j \mapsto \begin{cases}j & \text { if } j<p \\ ((j-p) \bmod q)+p & \text { if } j \geq p,\end{cases}
$$

so that $R_{j, i}$ and $R_{j \pi, i}$ are equal for all $i \in I$ and $j \in \mathbb{N}$.

The relation

$$
\begin{aligned}
F_{k} & =\left\{\left(a^{\beta}, a^{\gamma}\right): \beta, \gamma \in \mathbb{N} \cup\{0\},\lfloor\beta / k\rfloor=\lfloor\gamma / k\rfloor\right\} \\
& =\left\{\left(a^{k \eta}, a^{k \eta}\right): \eta \in \mathbb{N} \cup\{0\}\right\}\left\{\left(a^{\mu}, a^{\nu}\right): \mu, \nu \in\{0, \ldots, k-1\}\right\}
\end{aligned}
$$


is also regular.

Furthermore,

$$
\begin{aligned}
& \Lambda(\circ, \phi) \\
& =\left\{\left(a^{\alpha}, a^{\beta}, a^{\gamma}\right): \alpha, \beta, \gamma \in \mathbb{N} \cup\{0\},\left(a^{\alpha} \phi\right)\left(a^{\beta} \phi\right)=\left(a^{\gamma} \phi\right)\right\} \\
& =\left\{\left(a^{\alpha}, a^{\beta}, a^{\gamma}\right): \alpha, \beta, \gamma \in \mathbb{N} \cup\{0\},\right. \\
& \left(\lfloor(\alpha \bmod k) / r\rfloor, t_{\alpha \bmod r},\lfloor\alpha / k\rfloor\right)\left(\lfloor(\beta \bmod k) / r\rfloor, t_{\beta \bmod r},\lfloor\beta / k\rfloor\right) \\
& \left.=\left(\lfloor(\gamma \bmod k) / r\rfloor, t_{\gamma \bmod r},\lfloor\gamma / k\rfloor\right)\right\} \\
& =\left\{\left(a^{\alpha}, a^{\beta}, a^{\gamma}\right): \alpha, \beta, \gamma \in \mathbb{N} \cup\{0\},\right. \\
& \left(\lfloor(\alpha \bmod k) / r\rfloor, t_{\alpha \bmod r} p_{\lfloor\alpha / k\rfloor,\lfloor(\beta \bmod k) / r\rfloor} t_{\beta \bmod r},\lfloor\beta / k\rfloor\right) \\
& \left.=\left(\lfloor(\gamma \bmod k) / r\rfloor, t_{\gamma \bmod r},\lfloor\gamma / k\rfloor\right)\right\} \\
& =\left\{\left(a^{\alpha}, a^{\beta}, a^{\gamma}\right): \alpha, \beta, \gamma \in \mathbb{N} \cup\{0\},\right. \\
& \lfloor(\alpha \bmod k) / r\rfloor=\lfloor(\gamma \bmod k) / r\rfloor \wedge\lfloor\beta / k\rfloor=\lfloor\gamma / k\rfloor \\
& \left.\wedge t_{\alpha \bmod r} p_{\lfloor\alpha / k\rfloor,\lfloor(\beta \bmod k) / r\rfloor} t_{\beta \bmod r}=t_{\gamma \bmod r}\right\} \\
& =\left\{\left(a^{\alpha}, a^{\beta}, a^{\gamma}\right): \alpha, \beta, \gamma \in \mathbb{N} \cup\{0\},\right. \\
& \lfloor(\alpha \bmod k) / r\rfloor=\lfloor(\gamma \bmod k) / r\rfloor \wedge\lfloor\beta / k\rfloor=\lfloor\gamma / k\rfloor \\
& \left.\wedge\left(a^{\alpha}, a^{\beta}, a^{\gamma}\right) \in R_{\lfloor\alpha / k\rfloor,\lfloor(\beta \bmod k) / r\rfloor}\right\} \\
& =\left\{\left(a^{\alpha}, a^{\beta}, a^{\gamma}\right): \alpha, \beta, \gamma \in \mathbb{N} \cup\{0\},\right. \\
& \lfloor(\alpha \bmod k) / r\rfloor=\lfloor(\gamma \bmod k) / r\rfloor \wedge\left(a^{\beta}, a^{\gamma}\right) \in F_{k} \\
& \left.\wedge\left(a^{\alpha}, a^{\beta}, a^{\gamma}\right) \in R_{\lfloor\alpha / k\rfloor \pi,\lfloor(\beta \bmod k) / r\rfloor}\right\} .
\end{aligned}
$$

The relations $R_{j, i}$ and $F_{k}$ are regular and an automaton can track integers modulo $k$ and modulo $p$ (the second being required by the definition of $\pi$ ). Thus the relation $\Lambda(\circ, \phi)$ is regular. Thus $\left(a^{*}, \phi\right)$ is a unary automatic presentation for $S$.

Proposition 37 does not extend to countable-by-countable Rees matrix semigroups as a consequence of Proposition 25, since if $G$ is a group, $\mathcal{M}[G ; I, J ; P]$ consists of a single $\mathcal{D}$-class, and the $\mathcal{R}$ - and $\mathcal{L}$-classes are respectively subsets of the form $\{i\} \times G \times J$ and $I \times G \times\{j\}$, for $i \in I$ and $j \in J$ (see [9, Sections 3.1-2]).

Since every completely simple semigroup is isomorphic to a Rees matrix semigroup over a group by the Rees-Suschkewitsch theorem [9, Theorem 3.3.1], Proposition 37 and the results of Section 8 yield a complete classification of unary FApresentable completely simple semigroups:

Theorem 38. A completely simple semigroup is unary FA-presentable if and only if it is either a finite semigroup or a finite-by-countable Rees matrix semigroup over a finite group.

Proof. First of all, let $S$ be a unary FA-presentable completely simple semigroup; the aim is to show that $S$ is of one of the two species given. Then $S=\mathcal{M}[G ; I, J ; P]$, where $G$ is a group and $P$ is a $J \times I$ matrix over $G$. By Proposition 27, the group 
$G$, being isomorphic to any $\mathcal{H}$-class of $S$, is finite. By Proposition 25, at least one of $I$ and $J$ is finite. Since $S$, like all FA-presentable structures, is either finite or countable, if one of $I$ or $J$ is infinite, it must be countable and so $S$ is a finite-bycountable Rees matrix semigroup over the finite group $G$. If both $I$ and $J$ are finite, then $S$ is finite.

Any finite semigroup is unary FA-presentable, and a finite-by-countable Rees matrix semigroup over a finite group is unary FA-presentable by Proposition 37 .

\subsection{Direct products}

If $G$ is the trivial group and $I$ and $J$ are countable, the Rees matrix semigroup $\mathcal{M}[G ; I, J ; P]$ is the countable-by-countable rectangular band, which is isomorphic to the direct product of a countable left zero semigroup and a countable right zero semigroup. Since countable left zero and right zero semigroups are unary FA-presentable Example 11, it follows that the class of unary FA-presentable semigroups is not closed under forming direct products. This contrasts the classes of general FA-presentable semigroups and general FA-presentable structures, both of which are closed under finite direct products [1, Corollary 5.2.6(i)]. However, the class of unary FA-presentable semigroups is closed under forming direct products with finite semigroups:

Proposition 39. A direct product of a unary FA-presentable semigroup and a finite semigroup is itself unary FA-presentable.

Proof. Suppose $S$ is a unary FA-presentable semigroup and $T$ is finite. If $S$ is finite, so is $S \times T$ and there is nothing to prove. So suppose $S$ admits an injective unary automatic presentation $\left(a^{*}, \phi\right)$. Suppose the elements of $T$ are $t_{0}, \ldots, t_{r-1}$.

Define a map

$$
\psi: b^{*} \rightarrow S \times T, \quad b^{\alpha} \mapsto\left(a^{\lfloor\alpha / r\rfloor} \phi, \alpha \bmod r\right) .
$$

Then $\psi$ is injective, so $\Lambda(=, \psi)=\left\{\left(b^{\alpha}, b^{\alpha}\right): \alpha \in \mathbb{N} \cup\{0\}\right\}$ is regular. Reasoning similar to the proof of Proposition 35 shows that $\Lambda(\circ, \psi)$ is regular. So $\left(b^{*}, \psi\right)$ is a unary automatic presentation for $S \times T$.

However, a direct product of two unary FA-presentable semigroups may be unary FA-presentable. For example, the direct product of two countable right zero semigroups is again a countable right zero semigroup.

Question 40. Given unary automatic presentations for two semigroups, is it decidable whether their direct product is unary FA-presentable?

\subsection{Free products}

The semigroup free product of two semigroups never satisfies a non-trivial semigroup identity, so by Theorem 21, no semigroup free product is unary FA-presentable. A 
monoid free product of two monoids only satisfies a non-trivial semigroup identity if and only if one of the monoids is trivial and the other monoid satisfies a nontrivial semigroup identity. In this case, the free product is isomorphic to the second monoid. Therefore, no non-trivial free products are unary FA-presentable, which is perhaps unsurprising given how restricted is the class of semigroup or monoid free products that admit general FA-presentations [4, Section 4].

\section{Acknowledgements}

The first author was supported by the project EP/C523229/1, funded by the EPSRC, and later by by the European Regional Development Fund through the programme COMPETE and by the Portuguese Government through the FCT (Fundação para a Ciência e a Tecnologia) under the project PEsTC/MAT/UI0144/2011 and through an FCT Ciência 2008 fellowship. The third author would like to thank Hilary Craig for all her help and encouragement.

\section{References}

[1] A. Blumensath. Automatic Structures. Diploma thesis, RWTH Aachen, 1999.

[2] A. J. Cain, G. Oliver, N. Ruškuc, and R. M. Thomas. Automatic presentations for cancellative semigroups. In Language and Automata Theory and Applications: Second International Conference, Tarragona, Spain, March 13-19, 2008, eds. C. Martín-Vide, H. Fernau, and F. Otto, Lecture Notes in Computer Science, vol. 5196 (Springer, 2008), pages 149-159.

[3] A. J. Cain, G. Oliver, N. Ruškuc, and R. M. Thomas. Automatic presentations for semigroups. Inform. and Comput., 207(11) (2009) 1156-1168.

[4] A. J. Cain, G. Oliver, N. Ruškuc, and R. M. Thomas. Automatic presentations and semigroup constructions. Theory Comput. Syst., 47(2) (2010) 568-592.

[5] A. H. Clifford. Naturally totally ordered commutative semigroups. Amer. J. Math., 76(1954) 631-646.

[6] A. H. Clifford and G. B. Preston. The Algebraic Theory of Semigroups (Vol. I). Mathematical Surveys, No. 7 (American Mathematical Society, 1961).

[7] C. Delhommé. Automaticité des ordinaux et des graphes homogènes. C. R. Math. Acad. Sci. Paris, 339(1) (2004) 5-10.

[8] J. E. Hopcroft and J. D. Ullman. Introduction to Automata Theory, Languages, and Computation. (Addison-Wesley Publishing Co., 1979).

[9] J. M. Howie. Fundamentals of Semigroup Theory, volume 12 of London Mathematical Society Monographs (New Series). (Clarendon Press, Oxford University Press, 1995).

[10] B. Khoussainov and A. Nerode. Automatic presentations of structures. In Logic and computational complexity (Indianapolis, IN, 1994), Lecture Notes in Computer Science, vol. 960 (Springer, 1995), pages 367-392.

[11] B. Khoussainov, A. Nies, S. Rubin, and F. Stephan. Automatic structures: richness and limitations. In Proceedings of the 19th IEEE Symposium on Logic in Computer Science (IEEE Computer Society, 2004), pages 110-119.

[12] J. Liu and M. Minnes. Deciding the isomorphism problem in classes of unary automatic structures. Theoret. Comput. Sci., 412(18) (2011) 1705-1717.

[13] A. Nies and R. M. Thomas. FA-presentable groups and rings. J. Algebra, 320(2) (2008) 569-585. 
[14] G. P. Oliver and R. M. Thomas. Automatic presentations for finitely generated groups. In 22nd Annual Symposium on Theoretical Aspects of Computer Science (STACS'05), Stuttgart, Germany, eds. V. Diekert and B. Durand, Lecture Notes in Comput. Sci., vol. 3404 (Springer, 2005), pages 693-704. 